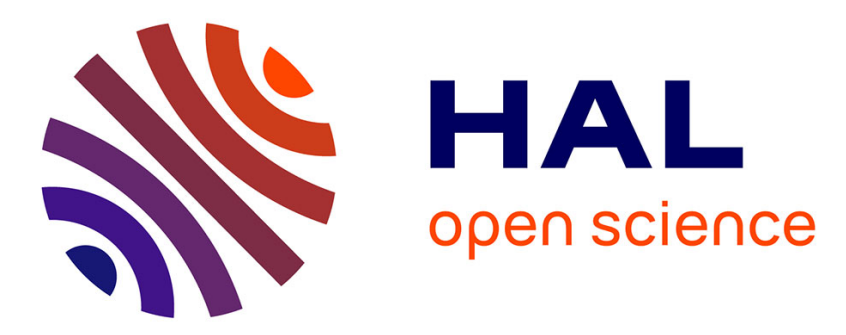

\title{
Influence of the analysis window on the metrological performance of the grid method
}

\author{
Frédéric Sur, Michel Grediac
}

\section{To cite this version:}

Frédéric Sur, Michel Grediac. Influence of the analysis window on the metrological performance of the grid method. Journal of Mathematical Imaging and Vision, 2016, 56 (3), pp.472-498. 10.1007/s10851016-0650-z . hal-01298523

\section{HAL Id: hal-01298523 \\ https://hal.inria.fr/hal-01298523}

Submitted on 6 Apr 2016

HAL is a multi-disciplinary open access archive for the deposit and dissemination of scientific research documents, whether they are published or not. The documents may come from teaching and research institutions in France or abroad, or from public or private research centers.
L'archive ouverte pluridisciplinaire HAL, est destinée au dépôt et à la diffusion de documents scientifiques de niveau recherche, publiés ou non, émanant des établissements d'enseignement et de recherche français ou étrangers, des laboratoires publics ou privés. 


\title{
Influence of the analysis window on the metrological performance of the grid method
}

\author{
Frédéric SuR*and Michel GrédiaC ${ }^{\dagger}$
}

\begin{abstract}
This paper deals with the grid method in experimental mechanics. It is one of the full-field methods available for estimating in-plane displacement and strain components of a specimen submitted to a load producing slight local deformation. This method consists in, first, depositing a regular grid on the surface of a specimen, and, second, comparing images of the grid before and after deformation. A possibility is to perform windowed Fourier analysis to measure these deformations as changes of the local grid aspect. The aim of the present study is to investigate the choice of the analysis window and its influence on the metrological performances of the grid method. Two aspects are taken into account, namely the reduction of the harmonics of the grid line profile, which are not pure sine because of manufacturing constraints, and the transfer of the digital noise from the imaged grid to the mechanical measurements. A theoretical study and a numerical assessment are presented. In addition, the interested reader can find in this paper a calculation of the Wigner-Ville transform of a triangular function, which, to the best of the present authors' knowledge, is not available in the existing literature.
\end{abstract}

Keywords: Image-based contactless measurement; displacement and strain maps; grid method; windowed Fourier analysis; spatially correlated noise; derivative of a random process; WignerVille transform

This is the author-manuscript version of

F. Sur, M. Grédiac. Influence of the analysis window on the metrological performance of the grid method. Journal of Mathematical Imaging and Vision, Springer, 2016.

DOI: $10.1007 / s 10851-016-0650-z$

${ }^{*}$ Université de Lorraine. LORIA UMR CNRS 7503, INRIA projet Magrit, Campus Scientifique, BP 239, 54506 Vandœuvre-lès-Nancy Cedex, France

${ }^{\dagger}$ Clermont Université, Université Blaise Pascal. Institut Pascal, UMR CNRS 6602, BP 10448, 63000 Clermont-Ferrand, France 


\section{Introduction}

\subsection{Motivation}

The windowed Fourier transform (WFT) is one of the tools available to process fringes in optics, or images of similar regular patterns like grids in experimental mechanics. Pseudo-periodic patterns being modeled as phase-modulated 2D periodic signals, the objective is to retrieve phase and phase derivatives, and ultimately displacement and strain distributions, as explained, for instance, in $[3,4,8,17,20,21,36]$. In this context, phase retrieval is somewhat a unique problem in the sense that the phase derivatives have tiny values with respect to the grid pattern frequency. The efficiency of the WFT is discussed in the aforementioned papers, but it seems that one of the parameters which influences the quality of the results, namely the very nature of the analysis window, has not really been studied in depth so far. In [21], it is proposed to employ a bidimensional Gaussian window on the ground that it reaches the lower bound provided by the uncertainty theorem [22]. However, it is also mentioned in [21] that "a simple square window also works quite well." In [3], the derivability of the Gaussian window is exploited to provide phase derivatives without calculating the phase itself. In [36], it is suggested to employ a triangular-rectangular window, and in [2] a bitriangular window is used, this window reducing the influence of the harmonics of the grid profile on the phase modulations which are identified. However, other $2 \mathrm{D}$ windows are used by some authors in discrete frameworks [34]. Note finally that the general question of the selection of the window is investigated in extensive review papers, for example, [14] or [26]. Concerning the grid method in experimental mechanics, the case of the Gaussian window has been extensively investigated in $[11,12,32]$.

In this context, the objective of this paper is to shed light on the choice of the analysis window employed in 2D WFT in the particular case of the grid method, which consists in retrieving displacement and strain maps from regular bidimensional grids slightly modulated by straining. This technique indeed raises an increasing interest in material and structure characterization $[5,9,27,29,30]$. In particular, the objective is generally to retrieve displacement and strain maps with the lowest random error and the smallest systematic errors as much as possible at the same time, which are contradictory requirements. Consequently, noise propagation between images of regular patterns and final maps must be thoroughly assessed along with the bias which impairs the measurements. Finally, since we deal here with full-field measurements, an additional metrological parameter must be considered: the spatial resolution. Although not clearly defined in metrology [19], it is obviously related here to the width of the analysis window. Spatial resolution is of prime importance to reliably distinguish close features in such maps. The reason is that when analyzing displacement and strain maps, the ultimate goal is to observe in detail the mechanical response of materials at the local level to understand and model subtle phenomena which occur at this scale, and establish the link with the macroscopic mechanical response.

Before specifying the aim of the proposed contribution, the next section gives some background information on the grid method. 

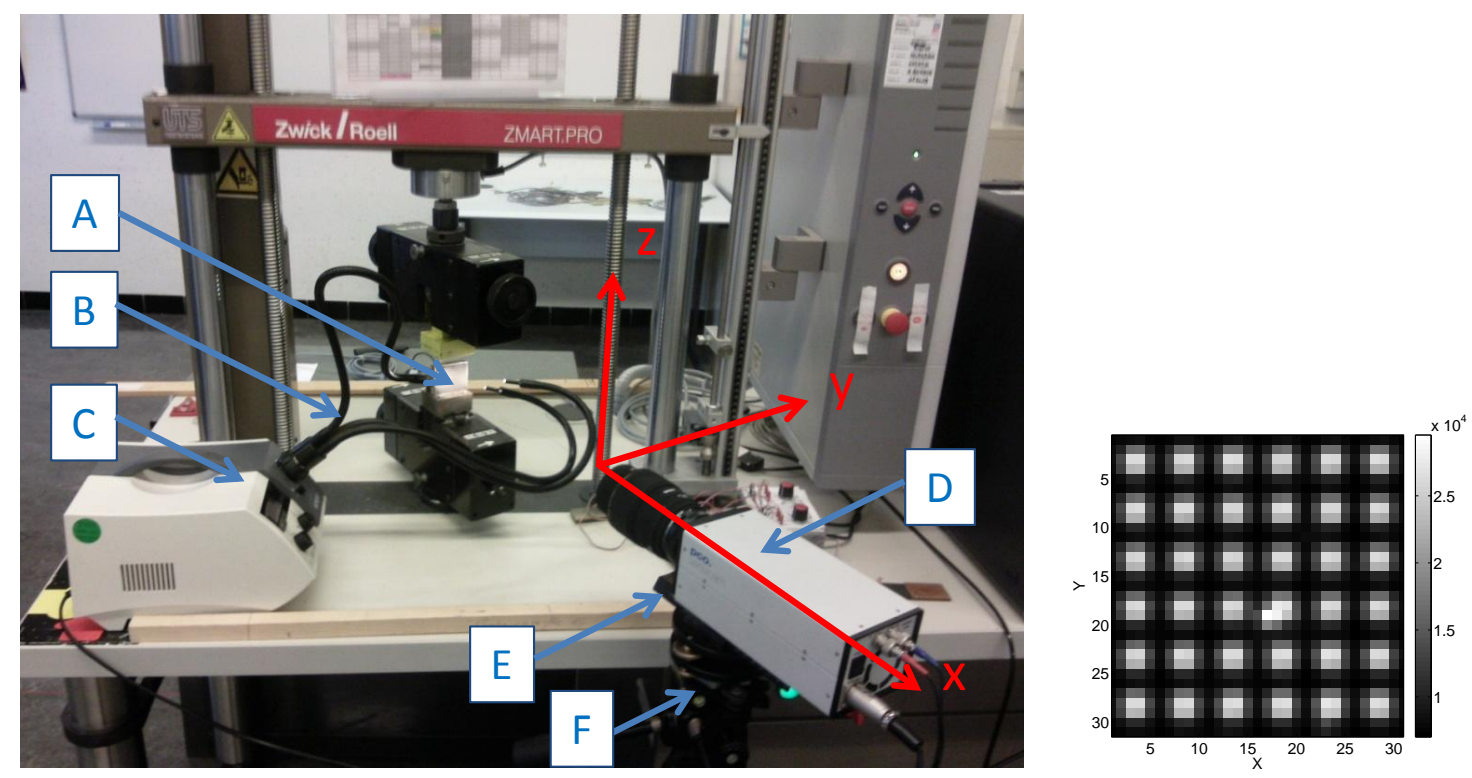

Figure 1: On the left: Typical experimental setup. A: camera; B: lighting; C: DC transformer; D: digital camera; E: two translation stages; F: three-way photographic head. On the right: close-up on a grid image.

\subsection{From grid images to displacement and strain maps}

This section explains how, in experimental mechanics, displacement and strain maps are retrieved from grid images. First, a pseudo-periodic, two-dimensional grid is deposited on the surface of a specimen to be analyzed. By "grid," we mean two orthogonal sets of parallel lines. For some practical reasons due to experimental setups which are generally employed when testing materials and structures (size of camera sensors, focal distance of typical lenses used for such experiments) and because of the area of the zones over which phenomena are generally expected to occur (typically some squared centimeters in most cases $[9,13,28])$, the distance between two consecutive lines is typically some tens of micrometers. This distance becomes much lower (some micrometers or less) when the surface is observed with a microscope [23, 24], or much greater when structural components are tested [30]. Note that the distance between two lines is usually limited to a few pixels in the images.

The specimen is subjected to a load, amplitude of which is measured, leading to surface deformation, thus grid modulation. It is then possible to retrieve displacement and strain maps from images of the grid taken before and after deformation. The experimental setup makes it possible to align the imaged grid to the pixel grid [12]. It is described in Figure 1.

The intensity at a pixel $\boldsymbol{x}=(x, y)$ of a grid image is thus modeled as (cf. [3])

$$
s(\boldsymbol{x})=A\left(1+\frac{\gamma}{2} \cdot \ell\left(2 \pi f x+\phi_{x}(\boldsymbol{x})\right)+\frac{\gamma}{2} \cdot \ell\left(2 \pi f y+\phi_{y}(\boldsymbol{x})\right)\right)+n(\boldsymbol{x})
$$


where

- $A>0$ is the global field illumination;

- $\gamma \in[0,1]$ is the contrast of the oscillatory pattern;

- the line profile $\ell$ is a $2 \pi$-periodic real function with a peak amplitude equal to 1 and average value 0 ;

- $f$ is the frequency of the carrier, $p=1 / f$ being the distance between any two consecutive lines;

- $\phi_{x}$ and $\phi_{y}$ are the carrier phase modulations due to specimen surface displacements and manufacturing variabilities along the $x$ and $y$-axes respectively. In practice, their spatial derivatives are very small with respect to $2 \pi f$ (typical values for the phase derivatives are smaller than $10^{-2}$ pixel $^{-1}$, while $1 / f=5$ pixels);

- $n$ is a noise term, inherent to any digital imaging device.

Although parameters $A$ and $\gamma$ may slightly vary in practice, they are assumed here to be constant. This feature has no deleterious effect since, as we shall see, the proposed estimation is based on local estimations.

Assuming that both phases $\phi_{x}$ and $\phi_{y}$ have been estimated before and after deformation $\left(\boldsymbol{\Phi}^{1}=\left(\phi_{x}^{1}, \phi_{y}^{1}\right)\right.$ and $\boldsymbol{\Phi}^{2}=\left(\phi_{x}^{2}, \phi_{y}^{2}\right)$ denoting the phase map pairs before and after deformation, respectively), it is possible to show that the displacement map $\boldsymbol{u}$ is the solution of the equation

$$
\boldsymbol{u}(\boldsymbol{x})=-\frac{p}{2 \pi}\left(\boldsymbol{\Phi}^{2}(\boldsymbol{x}+\boldsymbol{u}(\boldsymbol{x}))-\boldsymbol{\Phi}^{1}(\boldsymbol{x})\right)
$$

with $\boldsymbol{x}=(x, y)$, cf. [12].

This equation is solved with a few iterations of a fixed-point algorithm. The components of the linear strain tensor, which are the ultimate quantities of interest, are afterward derived from the displacement components by numerical differentiation:

$$
\varepsilon_{i j}=\frac{1}{2}\left(\frac{\partial u_{i}}{\partial j}+\frac{\partial u_{j}}{\partial i}\right) \quad \text { for } i, j=x, y
$$

The reader is invited to consult the recent review paper [12] on this technique for further details. In this paper, it is proved that, under the small displacement and strain hypothesis, we have

$$
\boldsymbol{u}(\boldsymbol{x}) \simeq \frac{p}{2 \pi}\left(\boldsymbol{\Phi}^{2}(\boldsymbol{x})-\boldsymbol{\Phi}^{1}(\boldsymbol{x})\right)
$$


and

$$
\varepsilon_{i j}(\boldsymbol{x}) \simeq \frac{p}{4 \pi}\left(\frac{\partial \phi_{i}^{2}}{\partial j}(\boldsymbol{x}+\boldsymbol{u}(\boldsymbol{x}))-\frac{\partial \phi_{i}^{1}}{\partial j}(\boldsymbol{x})+\frac{\partial \phi_{j}^{2}}{\partial i}(\boldsymbol{x}+\boldsymbol{u}(\boldsymbol{x}))-\frac{\partial \phi_{j}^{1}}{\partial i}(\boldsymbol{x})\right)
$$

for $i, j=x, y$.

We can see that estimating the phases and their derivatives is a cornerstone of the grid method to estimate displacement and strain maps. We investigate here a method based on the windowed Fourier transform, localized (in the Fourier domain) on the nominal value of the grid frequency: $(f, 0)$ along the $x$-axis and $(0, f)$ along the $y$-axis. This method consists in first calculating the following integral $[3,36]$ :

$$
\Psi(\boldsymbol{\xi}, \theta)=\iint_{\mathbb{R}^{2}} s(\boldsymbol{x}) w_{\boldsymbol{\sigma}}(\boldsymbol{x}-\boldsymbol{\xi}) e^{-2 i \pi f(x \cos (\theta)+y \sin (\theta))} \mathrm{d} \boldsymbol{x}
$$

where $\theta=0$ or $\theta=\pi / 2$, and $w_{\boldsymbol{\sigma}}$ is an analysis window of width $\boldsymbol{\sigma}$ (to be defined later). This window is symmetric along both axes, positive, and integrates to 1 , but it is not necessarily isotropic. An estimation of $\phi_{x}$ (resp. $\phi_{y}$ ) is then provided by $\arg (\Psi)$ with $\theta=0$ (resp. with $\theta=\pi / 2$ ), up to an additive constant. More precisely, the following first-order approximations were demonstrated in [32] under the small strain hypothesis:

$$
\left\{\begin{array}{l}
\arg \left((\Psi(\boldsymbol{\xi}, 0)) \simeq \arg \left(d_{1}\right)+g_{\sigma} * \phi_{x}(\boldsymbol{\xi})+\widehat{n}_{1}(\boldsymbol{\xi})\right. \\
\arg \left((\Psi(\boldsymbol{\xi}, \pi / 2)) \simeq \arg \left(d_{1}\right)+g_{\sigma} * \phi_{y}(\boldsymbol{\xi})+\widehat{n}_{2}(\boldsymbol{\xi})\right.
\end{array}\right.
$$

where $d_{1}$ is the Fourier coefficient of the primary frequency of $\ell, \widehat{n}_{1}$ and $\widehat{n}_{2}$ are noise terms caused by sensor noise in the original grid image, and $*$ denotes the convolution product.

In the remainder of this paper, and without loss of generality, we focus on the $x$-axis, so $\theta=0$ in (6), that is, on the estimation of $\phi_{x}$.

\subsection{Proposed contribution}

Other full-field measuring techniques rely on regular pattern processing like fringes obtained with interferometric setups [18, 21,36].

The grid method differs from these techniques in different ways:

- this is a white-light technique, so neither laser nor optical component like beamsplitters, mirrors, or magnifying lenses are required;

- the pattern is of higher frequency than that of classic fringe distributions in an interferogram, the number of lines being generally greater than the number of fringes obtained in classic interferograms, say some hundreds in an image;

- the frequencies of the regular pattern (one along $x$, another along $y$ ) remain very close to the nominal frequency of the grid even after deformation, so that the impact of deformation can be regarded as slight phase variation; 
- the interest of this measuring technique is its ability to measure, within certain limits, slight phase changes and slight phase derivative changes between images, and consequently small displacements and strains. For the application fields considered here, namely mechanics of materials and structures, it is of prime importance to measure reliably such small quantities because most of the engineering materials only slightly deform when they are loaded. For instance, no movement is detectable to the naked eye between images of reference and current (under load) configurations. Retrieving quantitative information from such images is a topical issue, the main challenges being to detect at any pixel the smallest displacement and/or strain as possible between grid images, but often over the smallest areas (to detect localized effects which generally occur in engineering materials, for instance close to failure), and with the smallest systematic and random errors.

In this context, we propose to discuss the influence of the analysis window through three important parameters governing the metrological performances of the grid method, namely, measurement bias, spatial resolution, and measurement resolution. In Section 2 we recall some basic results from [32] and, in particular, we explore the influence of the analysis window on harmonics attenuation in phase and phase derivative maps, as well as on the measurement bias and the spatial resolution. Section 3 is devoted to the propagation of the noise inherent to any digital acquisition device, from the grid images to the displacement and strain maps. We generalize results from [32] to show that noise affecting these maps is spatially correlated, and we discuss the impact on the measurement resolution. Numerical and real experiments illustrate the theoretical results. Appendices present technical details, among which one can find the calculation of the Wigner-Ville transforms of the rectangular, triangular, and Gaussian functions.

\subsection{Principal notations and reminder}

In this paper, 2D-vectors are given in bold letters, as in the preceding paragraphs.

The Fourier transform of any integrable 2D function $f(\boldsymbol{x})$ is denoted by $\widehat{f}(\boldsymbol{u})$ and is defined as

$$
\widehat{f}(\boldsymbol{u})=\iint_{\mathbb{R}^{2}} f(\boldsymbol{x}) e^{-2 i \pi<\boldsymbol{x} \cdot \boldsymbol{u}>} \mathrm{d} \boldsymbol{x}
$$

where $\langle\boldsymbol{x} \cdot \boldsymbol{u}\rangle=x u+y v$ denotes the canonical scalar product. We also write the Fourier transform of a 1D function $f(x)$ as

$$
\widehat{f}(u)=\int_{\mathbb{R}} f(x) e^{-2 i \pi x u} \mathrm{~d} x
$$

Let us recall that the value of the Fourier transform of $f$ at the origin is the mean value of $f$, and that the Fourier transform of a real symmetric function is also a real symmetric function.

We denote by $\mathbb{Z}$ the set of integers, by $\nabla \phi$ the gradient of any derivable function $\phi$, and by $\|\cdot\|_{2}$ the Euclidean norm in $\mathbb{R}^{2}$. 
The modulus of any complex number $z$ is denoted by $|z|$, and if $z \neq 0$, its argument is denoted by $\arg (z)$. Any equality on arguments is implicitly supposed to hold modulo $2 \pi$.

The cardinal sine function is denoted by sinc. For any $x \neq 0, \operatorname{sinc}(x)=\sin (\pi x) /(\pi x)$ and $\operatorname{sinc}(0)=1$. We recall that for any non-zero integer $n$ and a small $\varepsilon$, a first-order approximation gives

$$
\operatorname{sinc}(n+\varepsilon) \simeq(-1)^{n} \frac{\varepsilon}{n}
$$

The indicator function of any interval $[-a, a](a>0)$ is denoted by $\mathbb{1}_{[-a, a]}$. It is defined as $\mathbb{1}_{[-a, a]}(x)=1$ if $-a \leq x \leq a$ and $\mathbb{1}_{[-a, a]}(x)=0$ otherwise.

The covariance of two random variables $X$ and $Y$ is denoted by $\operatorname{Cov}(X, Y)$, the variance of $X$ being $\operatorname{Var}(X)$.

When visually representing a $2 \mathrm{D}$ discrete function $f(x, y)$ as the intensity of an image, $x$ denotes the row number and $y$ denotes the column number.

\section{Influence of the analysis window on the retrieved phase}

The goal of this section is to explore to which extent the choice of the analysis window $w_{\boldsymbol{\sigma}}$ influences the estimation of the phase as the argument of $\Psi$. As we shall see in Section 2.1, the analysis window must be carefully tuned in order to smooth out the harmonics of the line profile, so that they do not spoil the retrieved phases. The noise term in (1) is not considered in this section, it is the subject of Section 3.

\subsection{Phase estimation with windowed Fourier analysis}

All of the analysis windows which are actually used are separable. That is, they are the product of two $1 \mathrm{D}$ windows $w_{\sigma_{x}}^{x}$ and $w_{\sigma_{y}}^{y}$ of respective width $\sigma_{x}$ and $\sigma_{y}$ (such that $\boldsymbol{\sigma}$ denotes $\left.\left(\sigma_{x}, \sigma_{y}\right)\right)$, i.e., $w_{\boldsymbol{\sigma}}(x, y)=w_{\sigma_{x}}^{x}(x) w_{\sigma_{y}}^{y}(y)$. The Fourier transform of $w_{\boldsymbol{\sigma}}$ is thus also separable, thus $\widehat{w_{\boldsymbol{\sigma}}}(\xi, \eta)=\widehat{w_{\sigma_{x}}^{x}}(\xi) \widehat{w_{\sigma_{y}}^{y}}(\eta)$ holds.

Let us introduce some notations. Following [32], we introduce:

$$
\begin{gathered}
I_{1}(\boldsymbol{\xi})=\iint w_{\boldsymbol{\sigma}}(\boldsymbol{x}-\boldsymbol{\xi}) e^{-i 2 \pi f x} \mathrm{~d} \boldsymbol{x} \\
I_{2}(\boldsymbol{\xi})=\iint \ell\left(2 \pi f x+\phi_{x}(\boldsymbol{x})\right) w_{\boldsymbol{\sigma}}(\boldsymbol{x}-\boldsymbol{\xi}) e^{-i 2 \pi f x} \mathrm{~d} \boldsymbol{x} \\
I_{3}(\boldsymbol{\xi})=\iint \ell\left(2 \pi f y+\phi_{y}(\boldsymbol{x})\right) w_{\boldsymbol{\sigma}}(\boldsymbol{x}-\boldsymbol{\xi}) e^{-i 2 \pi f x} \mathrm{~d} \boldsymbol{x}
\end{gathered}
$$

so that:

$$
\Psi(\boldsymbol{\xi}, 0)=A I_{1}(\boldsymbol{\xi})+\frac{\gamma A}{2} I_{2}(\boldsymbol{\xi})+\frac{\gamma A}{2} I_{3}(\boldsymbol{\xi}) .
$$

Since $\ell$ is a 0 -mean $2 \pi$-periodic function, its Fourier series writes:

$$
\ell(x)=\sum_{k \in \mathbb{Z}} d_{k} e^{i k x}
$$


where $d_{0}=0$ and the $d_{k}$ are complex numbers. We also define $D=\left|\sum_{k \in \mathbb{Z}} k d_{k}\right|$, which is finite as soon as $\ell$ is smooth enough, for instance of class $C^{3}$.

Let us define for any $C^{2}$ function $\phi$ from $\mathbb{R}^{2}$ to $\mathbb{R}$ :

$$
M_{\boldsymbol{\sigma}}(\phi)(\boldsymbol{\xi})=\frac{1}{2} \iint\left|\boldsymbol{x} \mathcal{H}_{\boldsymbol{x}}^{\boldsymbol{\xi}} \boldsymbol{x}^{T}\right| \cdot w_{\boldsymbol{\sigma}}(\boldsymbol{x}) \mathrm{d} \boldsymbol{x}
$$

where $\mathcal{H}_{x}^{\xi}$ is a $2 \times 2$ matrix whose coefficients bound from above in $[\xi, \xi+x] \times[\eta, \eta+y]$ the coefficients of the Hessian matrix of $\phi$ at $\boldsymbol{\xi}$.

We can see that $M_{\boldsymbol{\sigma}}(\phi)$ is a weighted norm, localized around $(x, y)=(0,0)$, depending on the width of the analysis window.

The following theorem is a reminder of Theorem 2.1 in [32]. It permits us to further simplify each of the $I_{1}, I_{2}$, and $I_{3}$ integrals, and to emphasize the link between the Fourier transform of the analysis window $w_{\boldsymbol{\sigma}}$ and the Fourier series expansion of the grid line profile.

Theorem 1 The three following relations hold:

$$
\begin{gathered}
\left|I_{1}(\boldsymbol{\xi})\right|=\left|\widehat{w_{\boldsymbol{\sigma}}}(f, 0)\right| \\
\left|I_{3}(\boldsymbol{\xi})\right| \leq \sum_{k \in \mathbb{Z} \backslash\{0\}}\left|d_{k}\right| \widehat{w_{\boldsymbol{\sigma}}}\left(f-\frac{k}{2 \pi} \frac{\partial \phi_{y}}{\partial \xi}(\boldsymbol{\xi}), f k-\frac{k}{2 \pi} \frac{\partial \phi_{y}}{\partial \eta}(\boldsymbol{\xi})\right)+D \cdot M_{\boldsymbol{\sigma}}\left(\phi_{y}\right)(\boldsymbol{\xi}) \\
I_{2}(\boldsymbol{\xi})=d_{1} \iint w_{\boldsymbol{\sigma}}(\boldsymbol{x}-\boldsymbol{\xi}) e^{i \phi_{x}(\boldsymbol{x})} d \boldsymbol{x}+I_{2}^{\prime}(\boldsymbol{\xi})
\end{gathered}
$$

where:

$$
\left|I_{2}^{\prime}(\boldsymbol{\xi})\right| \leq \sum_{k \neq 0,1}\left|d_{k}\right| \cdot \widehat{w_{\boldsymbol{\sigma}}}\left((1-k) f-\frac{k}{2 \pi} \frac{\partial \phi_{x}}{\partial \xi}(\boldsymbol{\xi}), \frac{k}{2 \pi} \frac{\partial \phi_{x}}{\partial \eta}(\boldsymbol{\xi})\right)+D \cdot M_{\boldsymbol{\sigma}}\left(\phi_{x}\right)(\boldsymbol{\xi})
$$

This theorem states that, if integrals $I_{1}, I_{2}^{\prime}$, and $I_{3}$ can be neglected, then the argument of $\Psi$ is simply $\arg \left(d_{1}\right)+\arg \left(\iint w_{\boldsymbol{\sigma}}(x-\xi, y-\eta) e^{i \phi_{x}(x, y)} \mathrm{d} x \mathrm{~d} y\right)$. When the argument $\phi_{x}$ is properly unwrapped, $\arg (\Psi)$ turns out to be well approximated by $\arg \left(d_{1}\right)+$ $w_{\boldsymbol{\sigma}} * \phi_{x}[32]$.

On the one hand, if these terms cannot be neglected, the argument of $\Psi$ is affected by terms depending on the harmonics of the line profile $\ell$ (as can be seen from $I_{2}^{\prime}$ and $I_{3}$ ), and by the argument of $I_{1}$ which writes

$$
\arg \left(I_{1}(\xi, \eta)\right)=2 \pi f \xi
$$

because $I_{1}=e^{2 i \pi f \xi} \widehat{w_{\boldsymbol{\sigma}}}$ and $\widehat{w_{\boldsymbol{\sigma}}}$ is a real-valued function.

On the other hand, these terms can be neglected provided that:

- $\widehat{w_{\boldsymbol{\sigma}}}(f, 0)=\widehat{t_{\boldsymbol{\sigma}}^{\mathbf{x}}}(f)$ is negligible (from $(17)$ ); 
- The harmonics of the grid pattern (coefficients $d_{k}$ ) are discarded, that is, the corresponding weights $\widehat{w_{\boldsymbol{\sigma}}}(\cdot, \cdot)$ vanish in (18) and $(20)$;

- $M_{\boldsymbol{\sigma}}\left(\phi_{x}\right)$ and $M_{\boldsymbol{\sigma}}\left(\phi_{y}\right)$, that is, the second order terms in the sense of the weighted norm, are neglected.

Terms $M_{\boldsymbol{\sigma}}\left(\phi_{x}\right)$ and $M_{\boldsymbol{\sigma}}\left(\phi_{y}\right)$ depend on the behavior of the second-order phase derivatives inside the analysis window. The order of magnitude of these derivatives being very low in experimental mechanics applications, these terms can be confidently neglected, whatever the shape of the analysis window. As we shall see, this is not the case of the two first terms which involve the harmonics of the grid profile. Since the coefficients $d_{k}$ are likely to quickly decrease with $|k|$ as the Fourier coefficients of any smooth function, we are practically interested in canceling the influence of $\left|d_{k}\right|$ for small values of $|k|$. In practice, the grid is sampled with a few pixels per period (typically between 5 and 10 pixels), which means that $k$ is at most between 3 and 5 if the grid image is well sampled.

\subsection{Effect of the analysis window on harmonics attenuation}

As mentioned in the introduction, papers from the literature focus on three analysis window families, namely birectangular (product of two 1D rectangular windows), triangular-rectangular (product of a $1 \mathrm{D}$ triangle along $x$-direction and a $1 \mathrm{D}$ rectangle along $y$-direction, the window being rotated to calculate $\Psi(x, y, \pi / 2))$, bitriangular (product of two 1D triangular windows), and Gaussian windows. The 1D profiles of these windows are given in Table 1 and illustrated in Figure 2. It should be mentioned that the width of an analysis window has no univocal definition: for instance, the support of Gaussian windows is not compact. The comparison of the different windows in terms of spatial resolution is thus not obvious. Consequently, we will not keep the same notation for the parameter governing the window width, and we will argue about the comparisons in Section 2.4. From now on, $a, b$ and $\sigma$ are used for the parameters of a 1D rectangular, triangular, or Gaussian function, denoted by $r_{a}, t_{b}$, and $g_{\sigma}$, respectively. The parameters of the two $1 \mathrm{D}$ windows, product of which makes the $2 \mathrm{D}$ window, are potentially different.

As explained in the preceding section, we are interested in imposing that

- $\widehat{w_{\boldsymbol{\sigma}}}(f, 0)$,

- $\widehat{w_{\boldsymbol{\sigma}}}\left(f-\frac{k}{2 \pi} \frac{\partial \phi_{y}}{\partial \xi}(\xi, \eta),-f k+\frac{k}{2 \pi} \frac{\partial \phi_{y}}{\partial \eta}(\xi, \eta)\right)$ for $k \neq 0$,

- and $\left.\widehat{w_{\boldsymbol{\sigma}}}\left((k-1) f-\frac{k}{2 \pi} \frac{\partial \phi_{x}}{\partial \xi}(\xi, \eta),-\frac{k}{2 \pi} \frac{\partial \phi_{x}}{\partial \eta}(\xi, \eta)\right)\right)$ for $k \neq 0,1$

have the smallest possible values for "small" $k$, the phase derivatives being itself small with respect to $f$ in our applications.

The remainder of this section gives the values of these quantities in the cases of interest. 


\begin{tabular}{|c|c|c|}
\hline Window & Profile & Fourier transform \\
\hline Rectangular & $r_{a}(x)=\frac{1}{2 a} \mathbb{1}_{[-a, a]}(x)$ & $\widehat{r_{a}}(u)=\operatorname{sinc}(2 a u)$ \\
\hline Triangular & $t_{b}(x)=\frac{b-|x|}{b^{2}} \mathbb{1}_{[-b, b]}(x)$ & $\widehat{t_{b}}(u)=\operatorname{sinc}^{2}(b u)$ \\
\hline Gaussian & $g_{\sigma}(x)=\frac{1}{\sqrt{2 \pi} \sigma} e^{-x^{2} /\left(2 \sigma^{2}\right)}$ & $\widehat{g_{\sigma}}(u)=e^{-2 \pi^{2} \sigma^{2} u^{2}}$ \\
\hline
\end{tabular}

Table 1: Some popular 1D profiles and their Fourier transform. All of the profiles are symmetric and integrate to 1.
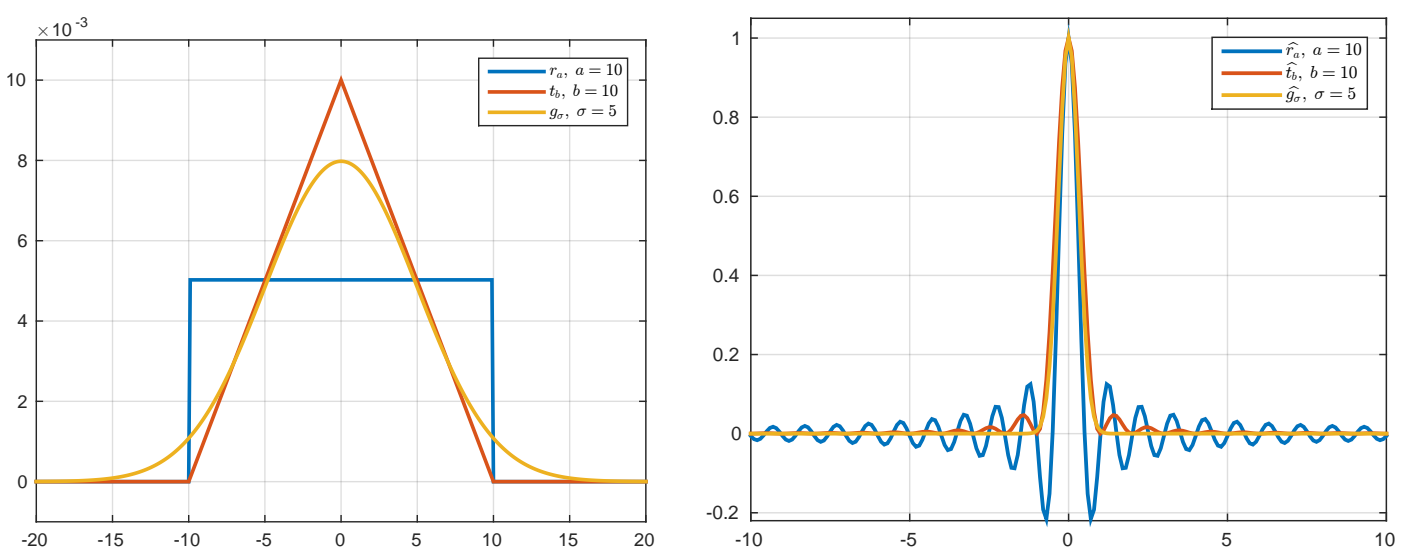

Figure 2: Graphical representation of the rectangular, triangular, and Gaussian profiles (on the left), and their respective Fourier transforms (on the right).

\subsubsection{Birectangular window}

In this case, the analysis window $w_{a_{1}, a_{2}}$ is the product of two rectangular functions, of width $2 a_{1}$ along the $x$-direction, and $2 a_{2}$ along the $y$-direction. With Table 1 ,

$$
\begin{aligned}
& \widehat{w_{a_{1}, a_{2}}}(f, 0)=\operatorname{sinc}\left(2 a_{1} f\right) \\
& \widehat{w_{a_{1}, a_{2}}}\left(f-\frac{k}{2 \pi} \frac{\partial \phi_{y}}{\partial \xi}(\boldsymbol{\xi}),-f k+\frac{k}{2 \pi} \frac{\partial \phi_{y}}{\partial \eta}(\boldsymbol{\xi})\right)=\operatorname{sinc}\left(2 a_{1} f-\frac{k a_{1}}{\pi} \frac{\partial \phi_{y}}{\partial \xi}(\boldsymbol{\xi})\right) \\
& \cdot \operatorname{sinc}\left(-2 a_{2} f k+\frac{k a_{2}}{\pi} \frac{\partial \phi_{y}}{\partial \eta}(\boldsymbol{\xi})\right)
\end{aligned}
$$




$$
\begin{array}{r}
\left.\widehat{w_{a_{1}, a_{2}}}\left((k-1) f-\frac{k}{2 \pi} \frac{\partial \phi_{x}}{\partial \xi}(\boldsymbol{\xi}),-\frac{k}{2 \pi} \frac{\partial \phi_{x}}{\partial \eta}(\boldsymbol{\xi})\right)\right)=\operatorname{sinc}\left(2(k-1) a_{1} f-\frac{k a_{1}}{\pi} \frac{\partial \phi_{x}}{\partial \xi}(\boldsymbol{\xi})\right) \\
\left.\cdot \operatorname{sinc}\left(-\frac{k a_{2}}{\pi} \frac{\partial \phi_{x}}{\partial \eta}(\boldsymbol{\xi})\right)\right)
\end{array}
$$

As we can see, (22) implies that $2 a_{1} f$ has a non-zero integer value, i.e., the parameter $a_{1}$ must be chosen such that $a_{1}=n /(2 f)$ for any non-zero integer $n$. If, in addition to this, $a_{2}$ is chosen such that $2 a_{2} f=m$ is an integer, the small derivative assumption and the first-order approximation given by (10) permit to approximate (23) and (24) by

$$
\begin{aligned}
& \widehat{w_{a_{1}, a_{2}}}\left(f-\frac{k}{2 \pi} \frac{\partial \phi_{y}}{\partial \xi}(\boldsymbol{\xi}),-f k+\frac{k}{2 \pi} \frac{\partial \phi_{y}}{\partial \eta}(\boldsymbol{\xi})\right) \simeq(-1)^{n+k m} \frac{k}{4 \pi^{2} f^{2}} \frac{\partial \phi_{y}}{\partial \xi}(\boldsymbol{\xi}) \frac{\partial \phi_{y}}{\partial \eta}(\boldsymbol{\xi}) \\
& \left.\widehat{w_{a_{1}, a_{2}}}\left((k-1) f-\frac{k}{2 \pi} \frac{\partial \phi_{x}}{\partial \xi}(\xi, \eta),-\frac{k}{2 \pi} \frac{\partial \phi_{x}}{\partial \eta}(\xi, \eta)\right)\right) \simeq \frac{-(-1)^{(k-1) n} k}{2 \pi(k-1) f} \frac{\partial \phi_{x}}{\partial \xi}(\xi, \eta)
\end{aligned}
$$

since $\operatorname{sinc}(\varepsilon) \simeq 1$ when $\varepsilon \simeq 0$.

Note that the quantities given by (23-24) are null only in the case of constant phases.

\subsubsection{Triangular-rectangular window}

The analysis window $w_{b, a}$ is the product of a triangular function of width $2 b$ along the $x$-direction and a rectangular function of width $2 a$ along the $y$-direction. With Table 1 ,

$$
\begin{array}{r}
\widehat{w_{b, a}}(f, 0)=\operatorname{sinc}^{2}(b f) \\
\widehat{w_{b, a}}\left(f-\frac{k}{2 \pi} \frac{\partial \phi_{y}}{\partial \xi}(\boldsymbol{\xi}),-f k+\frac{k}{2 \pi} \frac{\partial \phi_{y}}{\partial \eta}(\boldsymbol{\xi})\right)=\operatorname{sinc}^{2}\left(b f-\frac{k b}{2 \pi} \frac{\partial \phi_{y}}{\partial \xi}(\boldsymbol{\xi})\right) \\
\cdot \operatorname{sinc}\left(-2 a f k+\frac{k a}{\pi} \frac{\partial \phi_{y}}{\partial \eta}(\boldsymbol{\xi})\right) \\
\left.\widehat{w_{b, a}}\left((k-1) f-\frac{k}{2 \pi} \frac{\partial \phi_{x}}{\partial \xi}(\boldsymbol{\xi}),-\frac{k}{2 \pi} \frac{\partial \phi_{x}}{\partial \eta}(\boldsymbol{\xi})\right)\right)=\operatorname{sinc}^{2}\left((k-1) b f-\frac{k b}{2 \pi} \frac{\partial \phi_{x}}{\partial \xi}(\boldsymbol{\xi})\right) \\
\left.\cdot \operatorname{sinc}\left(-\frac{k a}{\pi} \frac{\partial \phi_{x}}{\partial \eta}(\boldsymbol{\xi})\right)\right)
\end{array}
$$

This time, (27) implies that $b f$ is a non-zero integer, i.e., $b=n / f$ is a multiple of the pattern pitch $p$. If, in addition to this, $2 a f=m$ is a non-zero integer, Equations (28) and (29) give:

$$
\begin{gathered}
\widehat{w_{b, a}}\left(f-\frac{k}{2 \pi} \frac{\partial \phi_{y}}{\partial \xi}(\boldsymbol{\xi}),-f k+\frac{k}{2 \pi} \frac{\partial \phi_{y}}{\partial \eta}(\boldsymbol{\xi})\right) \simeq \frac{(-1)^{m k+1} k^{2}}{4 \pi^{3} f^{3}}\left(\frac{\partial \phi_{y}}{\partial \xi}(\boldsymbol{\xi})\right)^{2} \frac{\partial \phi_{y}}{\partial \eta}(\boldsymbol{\xi}) \\
\left.\widehat{w_{b, a}}\left((k-1) f-\frac{k}{2 \pi} \frac{\partial \phi_{x}}{\partial \xi}(\boldsymbol{\xi}),-\frac{k}{2 \pi} \frac{\partial \phi_{x}}{\partial \eta}(\boldsymbol{\xi})\right)\right) \simeq \frac{k^{2}}{4 \pi^{2}(k-1)^{2} f^{2}}\left(\frac{\partial \phi_{x}}{\partial \xi}(\boldsymbol{\xi})\right)^{2}
\end{gathered}
$$

We can see that these error terms are an order of magnitude smaller than in the birectangular case, the derivative along $\xi$ being squared. 


\subsubsection{Bitriangular window}

In this case, the analysis window $w_{b_{1}, b_{2}}$ is the product of two triangular functions, of width $2 b_{1}$ along the $x$-direction and $2 b_{2}$ along the $y$-direction. With Table 1 ,

$$
\begin{array}{r}
\widehat{w_{b_{1}, b_{2}}}(f, 0)=\operatorname{sinc}^{2}(b f) \\
\widehat{w_{b_{1}, b_{2}}}\left(f-\frac{k}{2 \pi} \frac{\partial \phi_{y}}{\partial \xi}(\boldsymbol{\xi}),-f k+\frac{k}{2 \pi} \frac{\partial \phi_{y}}{\partial \eta}(\boldsymbol{\xi})\right)=\operatorname{sinc}^{2}\left(b_{1} f-\frac{k b_{1}}{2 \pi} \frac{\partial \phi_{y}}{\partial \xi}(\boldsymbol{\xi})\right) \\
\cdot \operatorname{sinc}^{2}\left(-b_{2} f k+\frac{k b_{2}}{2 \pi} \frac{\partial \phi_{y}}{\partial \eta}(\boldsymbol{\xi})\right) \\
\left.\widehat{w_{b_{1}, b_{2}}}\left((k-1) f-\frac{k}{2 \pi} \frac{\partial \phi_{x}}{\partial \xi}(\boldsymbol{\xi}),-\frac{k}{2 \pi} \frac{\partial \phi_{x}}{\partial \eta}(\boldsymbol{\xi})\right)\right)=\operatorname{sinc}^{2}\left((k-1) b_{1} f-\frac{k b_{1}}{2 \pi} \frac{\partial \phi_{x}}{\partial \xi}(\boldsymbol{\xi})\right) \\
\left.\cdot \operatorname{sinc}^{2}\left(-\frac{k b_{2}}{2 \pi} \frac{\partial \phi_{x}}{\partial \eta}(\boldsymbol{\xi})\right)\right)
\end{array}
$$

As in the preceding case, $b_{1} f$ must be an integer to cancel out (32). If $b_{2} f$ is also an integer,

$$
\begin{aligned}
& \widehat{w_{b_{1}, b_{2}}}\left(f-\frac{k}{2 \pi} \frac{\partial \phi_{y}}{\partial \xi}(\boldsymbol{\xi}),-f k+\frac{k}{2 \pi} \frac{\partial \phi_{y}}{\partial \eta}(\boldsymbol{\xi})\right) \simeq \frac{k^{2}}{16 \pi^{4} f^{4}}\left(\frac{\partial \phi_{y}}{\partial \xi}(\boldsymbol{\xi})\right)^{2}\left(\frac{\partial \phi_{y}}{\partial \eta}(\boldsymbol{\xi})\right)^{2} \\
& \left.\widehat{w_{b_{1}, b_{2}}}\left((k-1) f-\frac{k}{2 \pi} \frac{\partial \phi_{x}}{\partial \xi}(\boldsymbol{\xi}),-\frac{k}{2 \pi} \frac{\partial \phi_{x}}{\partial \eta}(\boldsymbol{\xi})\right)\right) \simeq \frac{k^{2}}{4 \pi^{2}(k-1)^{2} f^{2}}\left(\frac{\partial \phi_{x}}{\partial \xi}(\boldsymbol{\xi})\right)^{2}
\end{aligned}
$$

We can see that these error terms are still an order of magnitude smaller than in the triangular-rectangular case, the derivative along $\eta$ being squared.

\subsubsection{Gaussian window}

In this case, the analysis window is the product of two Gaussian functions, of standard deviation $\sigma_{x}$ along the $x$-direction and $\sigma_{y}$ along the $y$-direction. With Table 1 ,

$$
\begin{gathered}
\widehat{w_{\sigma_{x}}, \sigma_{y}}(f, 0)=e^{-2 \pi^{2} \sigma_{x}^{2} f^{2}} \\
\widehat{w_{\sigma_{x}, \sigma_{y}}}\left(f-\frac{k}{2 \pi} \frac{\partial \phi_{y}}{\partial \xi}(\boldsymbol{\xi}),-f k+\frac{k}{2 \pi} \frac{\partial \phi_{y}}{\partial \eta}(\boldsymbol{\xi})\right)=e^{-2 \pi^{2} \sigma_{x}^{2}\left(f-\frac{k}{2 \pi} \frac{\partial \phi_{y}}{\partial \xi}(\boldsymbol{\xi})\right)^{2}} \\
\cdot e^{-2 \pi^{2} \sigma_{y}^{2}\left(-f k+\frac{k}{2 \pi} \frac{\partial \phi_{y}}{\partial \eta}(\boldsymbol{\xi})\right)^{2}} \\
\left.\widehat{w_{\sigma_{x}, \sigma_{y}}}\left((k-1) f-\frac{k}{2 \pi} \frac{\partial \phi_{x}}{\partial \xi}(\boldsymbol{\xi}),-\frac{k}{2 \pi} \frac{\partial \phi_{x}}{\partial \eta}(\boldsymbol{\xi})\right)\right)=e^{-2 \pi^{2} \sigma_{x}^{2}\left((k-1) f-\frac{k}{2 \pi} \frac{\partial \phi_{y}}{\partial \xi}(\boldsymbol{\xi})\right)^{2}} \\
\cdot e^{-2 \pi^{2} \sigma_{y}^{2}\left(-\frac{k}{2 \pi} \frac{\partial \phi_{y}}{\partial \eta}(\boldsymbol{\xi})\right)^{2}}
\end{gathered}
$$


Contrary to the preceding windows, the Gaussian window does not need any condition on the $x$-direction width. It just needs $\sigma_{x} f$ to be large enough so that $e^{-2 \pi^{2} \sigma_{x}^{2} f^{2}}$ is negligible. Although $\sigma f \geq 1$ is sufficient in practice to satisfy this condition, we can note that this term does not rigorously cancel out.

Moreover, under the small derivative assumption

$$
\begin{gathered}
\widehat{w_{\sigma_{x}, \sigma_{y}}}\left(f-\frac{k}{2 \pi} \frac{\partial \phi_{y}}{\partial \xi}(\xi, \eta),-f k+\frac{k}{2 \pi} \frac{\partial \phi_{y}}{\partial \eta}(\xi, \eta)\right) \simeq e^{-2 \pi^{2} \sigma_{x}^{2} f^{2}} e^{-2 \pi^{2} \sigma_{y}^{2} f^{2} k^{2}} \\
\left.\widehat{w_{\sigma_{x}, \sigma_{y}}}\left((k-1) f-\frac{k}{2 \pi} \frac{\partial \phi_{x}}{\partial \xi}(\xi, \eta),-\frac{k}{2 \pi} \frac{\partial \phi_{x}}{\partial \eta}(\xi, \eta)\right)\right) \simeq e^{-2 \pi^{2} \sigma_{x}^{2}(k-1)^{2} f^{2}}
\end{gathered}
$$

We can note that these terms do not depend on the phase derivatives and are numerically negligible as soon as $\sigma_{x} f \geq 1$ and $\sigma_{y} f \geq 1$.

\subsubsection{Discussion}

Equations (22), (27), and (32) show that the window parameter should be a multiple of the pattern pitch $p=1 / f$ (triangular case) or of half of the pattern pitch (rectangular case) to avoid the influence of $I_{1}(\xi, \eta)$ on the phase, hence an additional $2 \pi f \xi$ term in the phase (see (21)). In the Gaussian case, this term, given by (37), cancels out as soon as the windows width is "large" with respect to $1 / f, \sigma$ larger than $1 / f$ being practically sufficient.

Under these conditions, the behavior with respect to the phase derivatives of, on the one hand, (25), (30), (35), (40), and, on the other hand, (26), (31), (36), (41), suggests that the Gaussian window should be the most able to cancel out the harmonics of the line profile, followed by the bitriangular, triangular-rectangular, and birectangular windows, in this order. It is also important to note that in the small derivative assumption, the attenuation of the harmonics does not depend on the phase derivatives in the Gaussian case, (40) and (41) having always negligible numerical values. This is not the case for bitriangular, triangular-rectangular, and birectangular windows, where the absolute values of (25-26), (30-31), and (35-36) increase with the phase derivatives.

In [36], it is advised to employ a triangular window with a width equal to twice the period of the pattern. Concerning the grid method, this advice is valid under the implicit assumption that the phases are constant inside the analysis window, as demonstrated by our calculations.

As a last comment, let us question the main assumption of the grid method, namely that the grid pattern is parallel to the sensor, with a pattern pitch $p=1 / f$ perfectly known. While it is practically a sound assumption, an imprecise experimental setting may yield a slight rotation of the specimen about any axis within the plane defined by the grid, leading to an out-of-plane displacement and making its surface not rigorously parallel to the image sensor. Denoting by $\delta_{p}$ an error on the pattern pitch, this gives an error $\delta_{f}$ on the frequency $f$ such that $p+\delta_{p}=1 /\left(f+\delta_{f}\right)$, thus a first-order expression $\delta_{f}=$ $-\delta_{p} / p^{2}$. As we can see from (1), an error $\delta_{f}$ on the frequency is equivalent to an additional $2 \pi \delta_{f} x$ term on the phase $\phi_{x}$, thus an additional $2 \pi \delta_{f}$ term on the phase derivatives. Since 
the alignment of the grid on the pixel rows and columns is assessed on a 1,000 pixel width image, an error of $\delta_{p}=0.001$ pixel is realistic. With a typical value of $p=5$ pixels, this gives $2 \pi \delta_{f}=-2 \pi \delta_{p} / p^{2}=-0.00025$ pixel $^{-1}$. This value is lower than usual phase derivatives. While this constant term vanishes in the phase difference used to estimate the strain component (see (5)), a larger $\delta_{p}$ error would prevent harmonics attenuation in the case of triangular or rectangular windows. Such an error could be also caused by geometric distortions induced by a cheap optical device, which would locally impair the grid alignment assumption. This property also advocates for the Gaussian window which does not require carefully setting its width.

\subsection{Illustrative numerical experiment}

While the theory of the preceding section was developed in the continuous case, we expect it to be still valid in the realistic discrete case. Moreover, the quantities of interest in experimental mechanics are not the phase and phase derivatives, but the displacement and strain maps. The goal of this section is to illustrate the theory by a numerical experiment. We generate 12 -bit 2,000 $\times 500$ synthetic grid images according to the model of (1), with:

- $A=2^{11}, \gamma=0.9, f=1 / 6$ pixel $^{-1}$;

- the line profile is the sine-cubed function (giving, with the notations of Section 2.1, $d_{1}=-d_{-1}=3 /(8 i), d_{3}=-d_{-3}=-1 /(8 i)$, and $d_{k}=0$ otherwise);

- $\phi_{x}$ and $\phi_{y}$ are equal to zero in the reference images, and, in the deformed image, given by the phase maps in Figure 3, normalized such that the phase derivatives (thus the strain maps) have a peak value of 0.005 .

The resulting gray value being quantized over 12 bits, some information is irremediably lost.

To assess the influence of the analysis window on displacement and strain map estimation, we estimate the difference between the retrieved maps $\widetilde{\mathcal{M}}(\mathbf{x})$ and the expected maps. Theorem 1 claims that the expected phases and phase derivatives are the convolution of the actual phases and phase derivatives by the analysis window, provided additional terms can be neglected. Under these conditions, the expected displacement and strain maps are approximately the actual maps $\mathcal{M}(\mathbf{x})$ convolved by the analysis window. We propose to measure the difference through the normalized root-mean-square error defined as

$$
\operatorname{NRMSE}(\sigma)=\frac{1}{N} \sqrt{\frac{1}{\# \Omega} \sum_{\mathbf{x} \in \Omega}\left|\widetilde{\mathcal{M}}(\mathbf{x})-g_{\sigma} * \mathcal{M}(\mathbf{x})\right|^{2}}
$$

where $\Omega$ is the pixel domain of the maps, $\# \Omega$ its cardinality, and the normalization term $N$ is given by

$$
N=\max _{\mathbf{x} \in \Omega}\left\{g_{\sigma} * \mathcal{M}(\mathbf{x})\right\}-\min _{\mathbf{x} \in \Omega}\left\{g_{\sigma} * \mathcal{M}(\mathbf{x})\right\}
$$



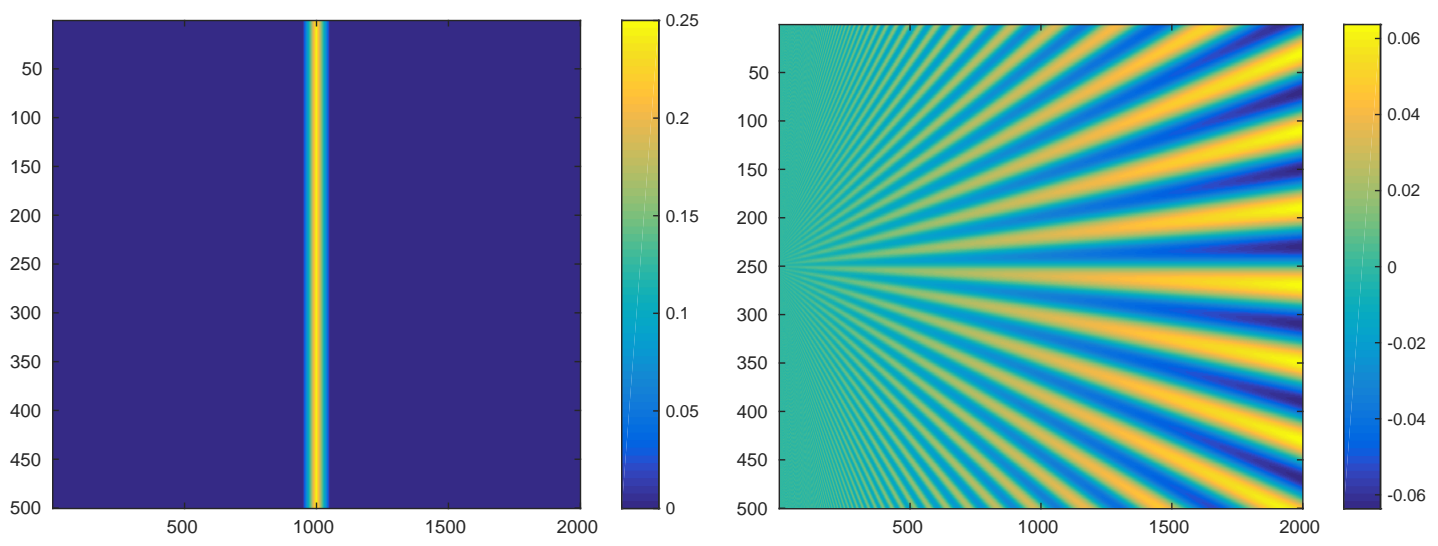

Figure 3: Synthetic phase maps for the deformed state. $\phi_{x}$ is a triangle function from $y=950$ to $y=1050$ of slope 0.005 , constant along the $x$-direction. $\phi_{y}$ is a made of a sine wave along the $x$-direction whose period linearly varies between 2 pixels at $y=1$ and 80 pixels at $y=2000$.

For the sake of simplicity, we test analysis windows of the same width in both directions, namely, with the notations of Table 1:

- birectangular window: $r_{a}(x) r_{a}(y)$

- triangular-rectangular windows: $t_{b}(x) r_{a}(y)$

- bitriangular window $t_{b}(x) t_{b}(y)$

- Gaussian window $g_{\sigma}(x) g_{\sigma}(y)$

with $a=b=2 \sigma$, this choice being motivated by the fact that $95 \%$ of the area below a Gaussian window is inside a $[-2 \sigma, 2 \sigma]$ interval. Note that this parameter setting does not lead to the same spatial resolution since we only focus here on harmonics attenuation, which is not an issue related to spatial resolution. This latter point is discussed further in the next section.

Figure 4 shows the evolution of the NRMSE as a function of $\sigma$, for each of the displacement and strain maps evaluated as explained in Section 1.2. The behavior of the NRMSE is as expected by the theory: it is approximately monotonous in the Gaussian case, and it has a periodic behavior in the other cases. Its minima are attained when $2 \sigma$ is a multiple of the pattern pitch (triangular and triangular-rectangular cases), or of half the pattern pitch (rectangular cases). We can see that the birectangular window performs much worse than the other windows, as expected from the discussion of Section 2.2.5. Triangular-rectangular and bitriangular windows give almost the same NRMSE (which is logical since the derivatives of $\phi_{x}$ are equal to zero outside a narrow band), except when estimating $\epsilon_{x y}$, where triangular-rectangular windows give a larger NRMSE because of 

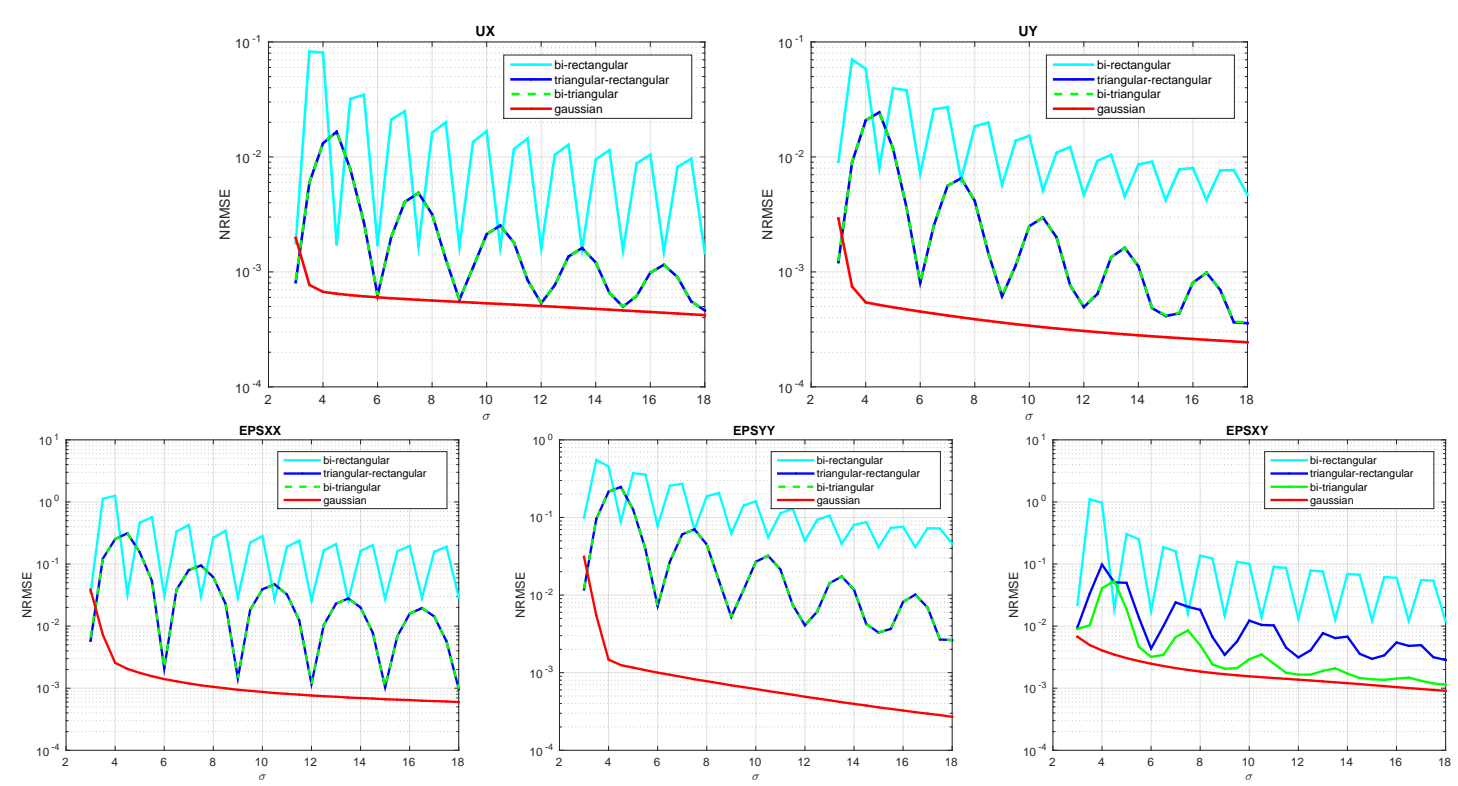

Figure 4: Normalized root-mean-square error for varying window sizes.

the cross derivatives. Gaussian windows always give smaller NRMSE, especially in the case of a non-localized displacement and strain maps such as $u_{y}$ and $\epsilon_{y y}$.

Even when the NRMSE is below $10 \%$, we can see in Figure 5 that periodic artifacts are potentially clearly visible in the retrieved maps. While the chosen window size corresponds to a minimum in the NRMSE of the birectangular window, artifacts can be seen. As expected, these artifacts are visually much more pronounced with the bitriangular window. They are not visible with the Gaussian window.

It is important to note that the goal here is not to retrieve the smallest NRMSE. The NRMSE indeed measures the difference between the retrieved maps and the ground truth convolved by the analysis windows. The decreasing of the NRMSE with the window width only depicts the decreasing of the relative error made when approximated the retrieved map by the convolution. However, the retrieved map is all the smoother as the window width is larger. A compromise must be made between smoothing and harmonics attenuation, that is why $\sigma$ is often chosen equal to the pattern pitch.

Another important point is that the performances of the windows cannot be directly compared. Because of their various shape, the $a=b=2 \sigma$ rule used for Figure 4 is very crude. The following section gives a comparison rule through the relative measurement bias and the spatial resolution of the method.

\subsection{Measurement bias and spatial resolution}

Instead of comparing the NRMSE of the different windows depending on an awkward parameter, we prefer to fix one of the metrological properties of the method. Following [37] 

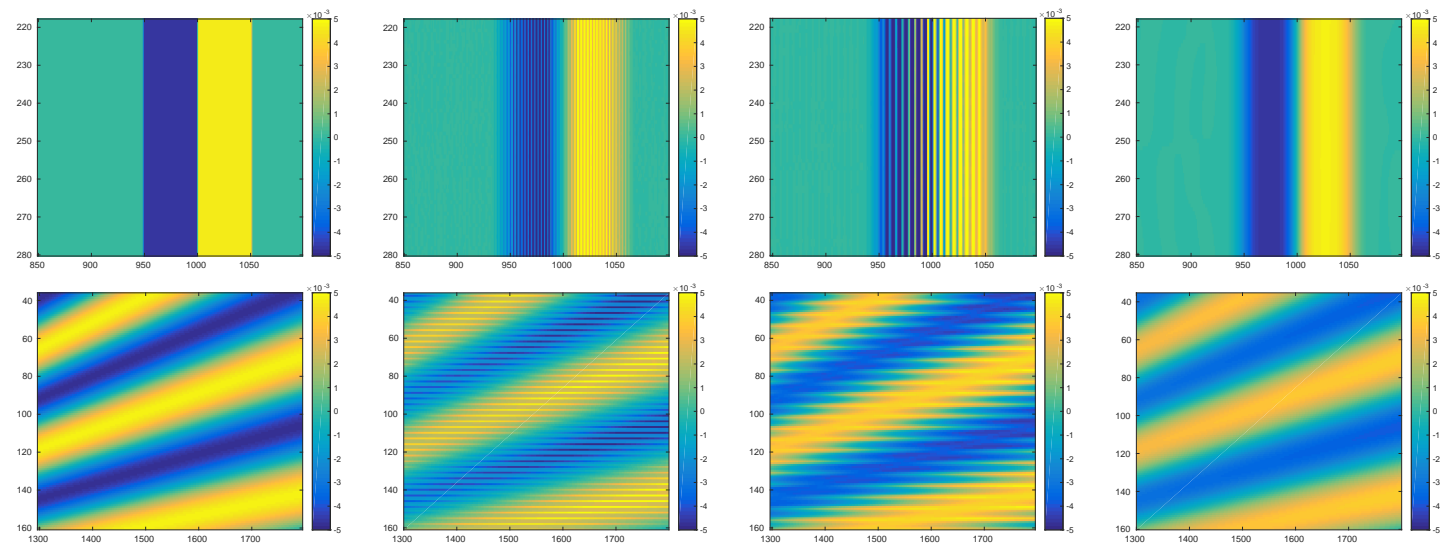

Figure 5: Close-up on some retrieved strain maps, with $\sigma=7.5$. Up: $\epsilon_{x x}$; Bottom: $\epsilon_{y y}$. From left to right: ground truth, birectangular window, bitriangular window, and Gaussian window.

(see also [12]), we define the spatial resolution as "the lowest period of a sinusoidal deformation that the technique is able to reproduce before losing a certain percentage of amplitude."

As recalled in the preceding section, the obtained phases and phase derivatives are approximately equal to the convolutions of the actual phase and the analysis window $w_{\boldsymbol{\sigma}}$. As the transfer function of any linear filter is the Fourier transform of its impulse response, a unitary sine wave $\sin _{f^{\prime}}$ of frequency $f^{\prime}$ along the $x$-direction is retrieved as a sine wave of the same frequency and of amplitude $\widehat{w_{\boldsymbol{\sigma}}}\left(f^{\prime}, 0\right)$. This is simply due to the following calculation:

$$
\begin{aligned}
w_{\boldsymbol{\sigma}} * \sin _{f^{\prime}}(u) & =\iint w_{\boldsymbol{\sigma}}(x, y) \frac{e^{2 i \pi f^{\prime}(u-x)}-e^{-2 i \pi f^{\prime}(u-x)}}{2 i} \mathrm{~d} x \mathrm{~d} y \\
& =\frac{1}{2 i}\left(e^{-2 i \pi f^{\prime} u} \widehat{w_{\boldsymbol{\sigma}}}\left(f^{\prime}, 0\right)-e^{2 i \pi f u} \widehat{w_{\boldsymbol{\sigma}}}\left(-f^{\prime}, 0\right)\right) \\
& =\widehat{w_{\boldsymbol{\sigma}}}\left(f^{\prime}, 0\right) \sin \left(2 \pi f^{\prime} u\right)
\end{aligned}
$$

Consequently, the relative measurement bias [12], defined as the ratio between the loss of amplitude and the amplitude of the reference sine function, writes:

$$
\lambda_{w_{\boldsymbol{\sigma}}}\left(f^{\prime}\right)=1-\left|\widehat{w_{\boldsymbol{\sigma}}}\left(f^{\prime}, 0\right)\right|
$$

We can see from the properties of the Fourier transform $\widehat{w_{\boldsymbol{\sigma}}}$ of any window that $\lambda_{w_{\boldsymbol{\sigma}}}\left(f^{\prime}\right) \in$ $[0,1], \quad \lambda_{w_{\boldsymbol{\sigma}}}(0)=0, \lambda_{w_{\boldsymbol{\sigma}}}\left(f^{\prime}\right)$ locally decreases around 0 , and $\lambda_{w_{\boldsymbol{\sigma}}}\left(f^{\prime}\right)$ tends to 1 when $f^{\prime}$ tends to $\infty$. This means that the relative measurement bias is barely noticeable in low-frequency components, and makes high-frequency components to vanish.

As an example, Figure 6 shows the retrieved phases for a synthetic grid image defined as in (1) with $\phi_{y}$ made of a sine wave along the $x$-direction whose period linearly varies 

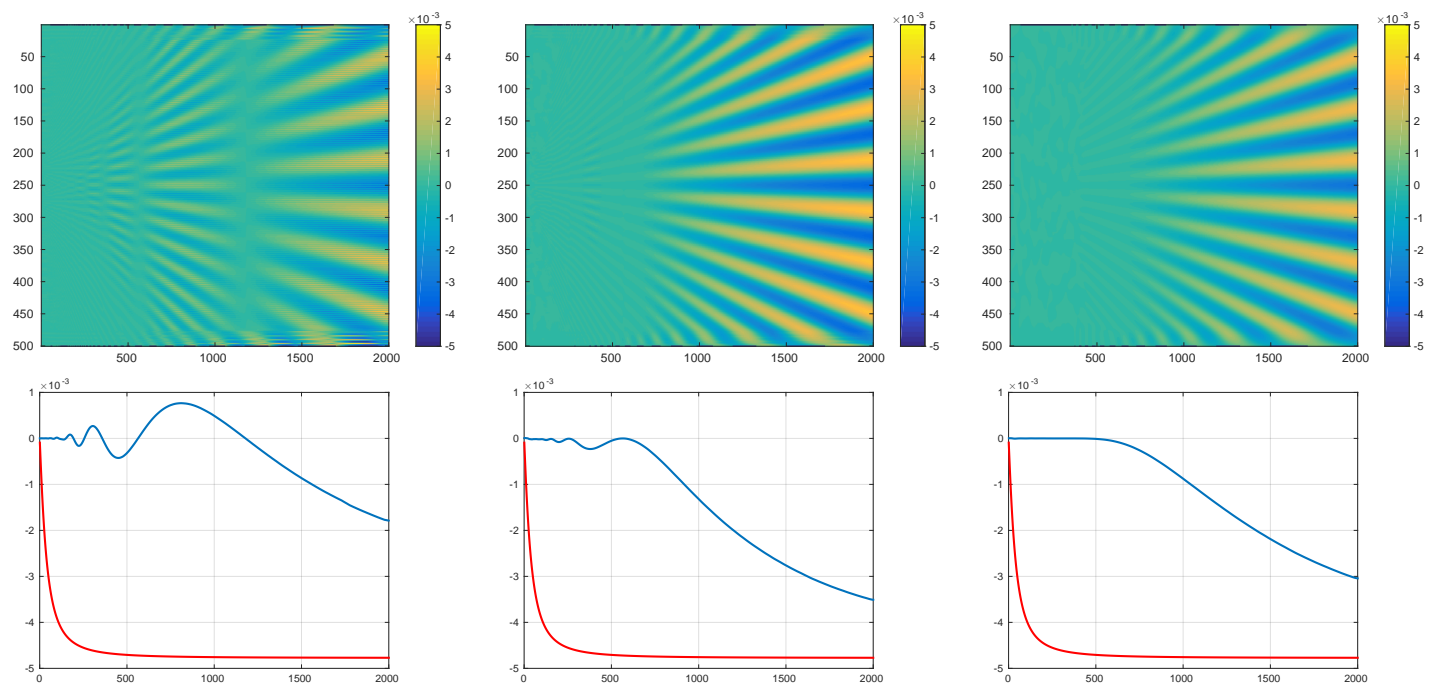

Figure 6: From left to right: retrieved strain component $\varepsilon_{y y}$ for birectangular $(a=24)$, bitriangular $(b=24)$, and Gaussian $\sigma=12$ windows, together with a cross-section at $x=250$ (in blue, the ground truth is in red).

between 2 pixels at $y=1$ and 80 pixels at $y=2000$. Three analysis windows are tested. As expected from the theory and the graphs of the attenuation functions, we can see that high-frequency components vanish. In the case of the birectangular window, it is also possible to see bands corresponding to the zeros of the cardinal sine (they are barely visible in the case of the bitriangular window, e.g., around $x=400-450)$, and the sign changes between them. This means that the sign of the measured strain components cannot even be trusted in the case of a rectangular window.

With the above-mentioned definition, the spatial resolution in the $x$-direction (as well as in the $y$-direction for symmetric windows) is:

$$
d_{w_{\boldsymbol{\sigma}}}=\inf \left\{p^{\prime}>0 \text {, such that } 1-\left|\widehat{w_{\sigma}}\left(1 / p^{\prime}, 0\right)\right|<\lambda^{*}\right\}
$$

where $\lambda^{*}$ is the reference bias. We note that $\widehat{w_{\sigma}}\left(1 / p^{\prime}, 0\right)$ is not monotonous in the triangular and rectangular cases, $1-\left|\widehat{w_{\sigma}}\left(1 / p^{\prime}, 0\right)\right|=\lambda^{*}$ having potentially several solutions $p$ for a large $\lambda^{*}$. In this paper, we set $\lambda^{*}=0.1$ which is below the local minima involved by the cardinal sine function for the typical window sizes.

Except for the Gaussian case (in which we find $d=\pi \sqrt{-2 / \log \left(1-\lambda^{*}\right)} \sigma$ ), no closedform expression can be found. A numerical estimation gives the following values of the spatial resolution for $\lambda^{*}=0.1$ :

- birectangular window $r_{a}(x) r_{a}(y): d=a / 0.1252$;

- triangular-rectangular window $t_{a}(x) r_{b}(y)$ : in the $x$-direction $d=a / 0.1780$ and in the $y$-direction $d=b / 0.1252$; 

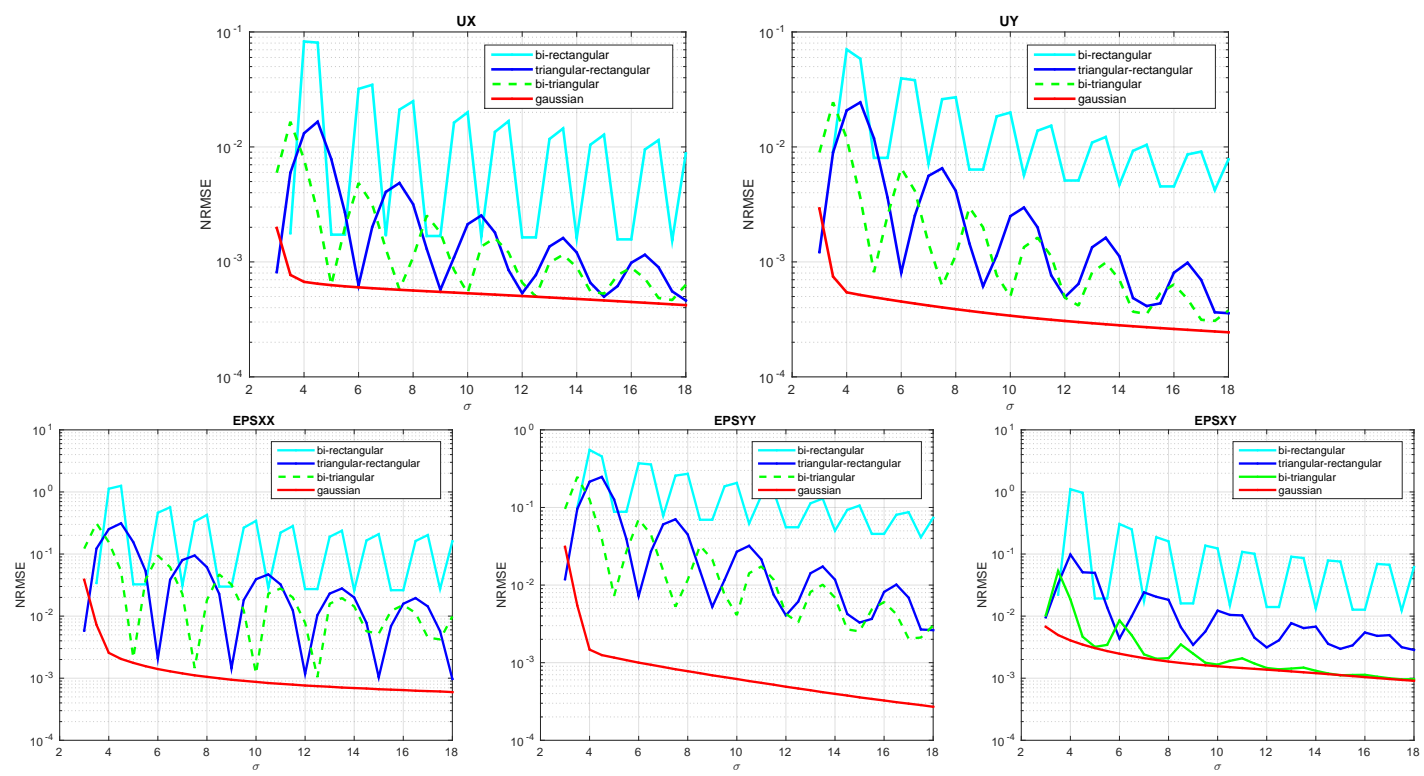

Figure 7: Normalized root-mean-square for varying window sizes chosen in such a way that the spatial resolution is, at a given $\sigma$ value, fixed in the four cases.

- bitriangular window $t_{b}(x) t_{b}(y): d=b / 0.1780$.

- Gaussian window $g_{\sigma}(x, y): d=\sigma / 0.0731$.

If the windows satisfy the relations $a=1.7137 \sigma$ and $b=2.4366 \sigma$, then the spatial resolutions of the birectangular, bitriangular, and Gaussian windows are identical. We overcome the difficulty with the non-symmetric triangular-rectangular window by imposing $a=b$ and $a b=1.7137 \sigma \times 2.4366 \sigma$, i.e., $a=b=2.0434 \sigma$.

With such a normalization, it is possible to compare the NRMSE curves at a fixed spatial resolution. The parameter of the Gaussian varies, and the $a$ and $b$ parameters are governed by the preceding relations. Since they have to be integer, we actually keep the nearest integer to the real value. This is illustrated in Figure 7 . We can see that the best compromise between spatial resolution and NRMSE is definitively attained by the Gaussian window.

As a last remark, we can note that the spatial resolution is, in general, not isotropic and actually depends on the direction of the considered sine wave. Equation (48) indeed extends to

$$
d_{w_{\boldsymbol{\sigma}}}(\theta)=\inf \left\{p^{\prime}>0 \text { such that } 1-\left|\widehat{w_{\sigma}}\left(\cos (\theta) / p^{\prime}, \sin (\theta)\right) / p^{\prime}\right|<\lambda^{*}\right\}
$$

with $\theta \in[0, \pi / 2]$ (which is sufficient because of the symmetry of $\widehat{w_{\sigma}}$ ) and a given $\lambda^{*}$, or equivalently, $d_{w_{\boldsymbol{\sigma}}}(\theta)=1 / f_{w_{\boldsymbol{\sigma}}}^{\prime}(\theta)$ where

$$
f_{w_{\boldsymbol{\sigma}}}^{\prime}(\theta)=\sup \left\{f^{\prime}>0 \text { such that } 1-\left|\widehat{w_{\sigma}}\left(f^{\prime} \cos (\theta), f^{\prime} \sin (\theta)\right)\right|<\lambda^{*}\right\}
$$



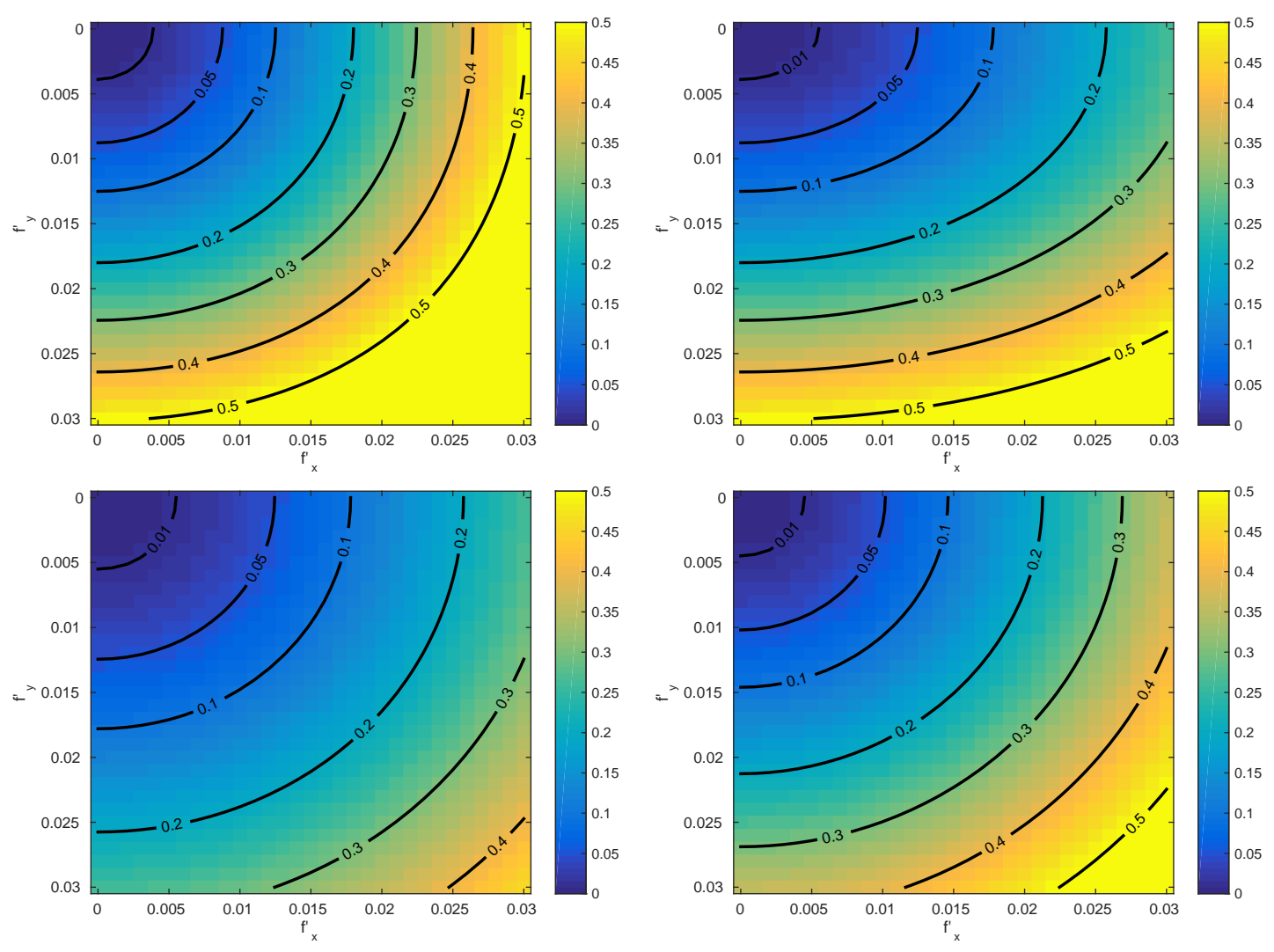

Figure 8: Map of $1-\widehat{w_{\sigma}}\left(f_{x}^{\prime}, f_{y}^{\prime}\right)$, and contour lines. From left to right and from top to bottom: birectangular window $r_{a}(x) r_{a}(y)$, triangular-rectangular window $t_{a}(x) r_{b}(y)$, bitriangular window $t_{b}(x) t_{b}(y)$, and Gaussian window $g_{\sigma}(x, y)$, with $a=b=10$ and $\sigma=$ 5 .

Among the considered windows, the Gaussian window is the only one which gives an isotropic spatial resolution.

As an illustration, Figure 8 shows the values taken by $1-\left|\widehat{w_{\sigma}}\left(f_{x}^{\prime}, f_{y}^{\prime}\right)\right|$ at $\left(f_{x}^{\prime}, f_{y}^{\prime}\right)$, for $r_{a}(x) r_{a}(y), t_{a}(x) r_{b}(y), t_{b}(x) t_{b}(y)$, and $g_{\sigma}(x, y)$, with $a=b=10$ and $\sigma=5$. Some contour lines are also shown. We can verify that the contour line $\lambda^{*}=0.1$ crosses the $x$ and $y$ axes in accordance with the previous numerical estimation: at $f_{x}^{\prime}=f_{y}^{\prime}=0.1252 / a$ for the birectangular windows, at $f_{x}^{\prime}=0.1252 / a$ and $f_{y}^{\prime}=0.1780 / b$ for the triangularrectangular window, at $f_{x}^{\prime}=f_{y}^{\prime}=0.1780 / b$ for the bitriangular window, and at $f_{x}^{\prime}=$ $f_{y}^{\prime}=0.0731 / \sigma$ for the Gaussian window. 


\section{Noise on the phases and on the phase derivatives}

This section deals with the propagation of the noise from the imaged grid to the phases and phase derivatives, and ultimately to the displacement and strain maps. The aim is to obtain closed-form expressions for the measurement resolution. We define here the measurement resolution as the standard deviation of the noise in the corresponding measurement map (phase, phase derivative, displacement or strain), as suggested in [6]. This quantity corresponds to the smallest change not likely to be caused by measurement noise, this noise being considered as due to camera sensor noise which propagates to the final maps. Raw images are actually affected by heteroscedastic, signal-dependent noise, and are usually modeled as a Poisson-Gaussian random field (see, e.g., [15]). Nevertheless, the generalized Anscombe transform [25] permits to transform these images into images impaired by a Gaussian white noise. While the effect of this transform on the retrieved displacement and strain maps still needs to be quantitatively assessed (numerical simulations seems to indicate that it is low), we have shown in [11] that this transform permits to experimentally assess measurement resolution expressions established under white noise assumption in the case of Gaussian windows in [32].

As a consequence, we assume that grid images are impaired by a white noise of variance $v$, even if it means that a variance stabilization transform has to be applied beforehand. The aim is to extend the results of [32] about noise propagation to other windows than Gaussian ones, in a framework that permits to make sound comparisons. As we shall see, displacement and strain maps are affected by a spatially correlated noise, whose covariance is calculated in this section. Note that, in the context of fringe analysis in optical interferometry, an analogous calculation of the variance is available in $[35,36]$, using a different approach and simplifying hypotheses.

\subsection{Effect of image noise on the windowed Fourier transform}

Since windowed Fourier transform is linear, a white noise $n$ (of variance $v$ ) affecting the grid image propagates to a complex noise $\widehat{n}$ on the windowed Fourier transform at $(f, 0)$, whose expression is:

$$
\widehat{n}(\xi, \eta)=\sum_{i, j} n\left(x_{i}, y_{j}\right) w_{\boldsymbol{\sigma}}\left(x_{i}-\xi, y_{j}-\eta\right) e^{-2 i \pi f x_{i}} \Delta_{x} \Delta_{y}
$$

where $\left(\Delta_{x}, \Delta_{y}\right)$ is the grid pitch in the image $s$ (here $\Delta_{x}=\Delta_{y}=1$ pixel). This means that, in (6), the discrete formulation of $\Psi$ is now impaired by the additive noise $\widehat{n}$.

Proposition 3.1 in [32] (proved by a calculation valid for Gaussian windows only) generalizes to the following proposition:

Proposition 1 The autocovariance functions of the real and imaginary parts of $\widehat{n}$ are 
given by

$$
\begin{array}{r}
\operatorname{Cov}\left(\operatorname{Re}(\widehat{n}(\xi, \eta)), \operatorname{Re}\left(\widehat{n}\left(\xi^{\prime}, \eta^{\prime}\right)\right)\right) \simeq \frac{v \Delta_{x} \Delta_{y}}{2} \\
\cdot\left(w_{\sigma_{x}}^{x} * w_{\sigma_{x}}^{x}\left(\xi-\xi^{\prime}\right)+\frac{1}{2} \mathcal{W} w_{\sigma_{x}}^{x}\left(\left(\xi-\xi^{\prime}\right) / 2,2 \pi f\right) \cos \left(2 \pi f\left(\xi+\xi^{\prime}\right)\right)\right) \\
\cdot w_{\sigma_{y}}^{y} * w_{\sigma_{y}}^{y}\left(\eta-\eta^{\prime}\right)
\end{array}
$$

$$
\begin{array}{r}
\operatorname{Cov}\left(\operatorname{Im}(\widehat{n}(\xi, \eta)), \operatorname{Im}\left(\widehat{n}\left(\xi^{\prime}, \eta^{\prime}\right)\right)\right) \simeq \frac{v \Delta_{x} \Delta_{y}}{2} \\
\cdot\left(w_{\sigma_{x}}^{x} * w_{\sigma_{x}}^{x}\left(\xi-\xi^{\prime}\right)-\frac{1}{2} \mathcal{W} w_{\sigma_{x}}^{x}\left(\left(\xi-\xi^{\prime}\right) / 2,2 \pi f\right) \cos \left(2 \pi f\left(\xi+\xi^{\prime}\right)\right)\right) \\
\cdot w_{\sigma_{y}}^{y} * w_{\sigma_{y}}^{y}\left(\eta-\eta^{\prime}\right)
\end{array}
$$

$$
\begin{aligned}
& \operatorname{Cov}\left(\operatorname{Re}(\widehat{n}(\xi, \eta)), \operatorname{Im}\left(\widehat{n}\left(\xi^{\prime}, \eta^{\prime}\right)\right)\right) \simeq \frac{v \Delta_{x} \Delta_{y}}{4} \\
& \cdot \mathcal{W} w_{\sigma_{x}}^{x}\left(\left(\xi-\xi^{\prime}\right) / 2,2 \pi f\right) \sin \left(2 \pi f\left(\xi+\xi^{\prime}\right)\right) \cdot w_{\sigma_{y}}^{y} * w_{\sigma_{y}}^{y}\left(\eta-\eta^{\prime}\right)
\end{aligned}
$$

where $\mathcal{W} w_{\sigma}$ is the Wigner-Ville transform ${ }^{1}$ of any $1 D$ window $w_{\sigma}$.

In this proposition, the approximations come from replacing discrete Riemann sums with the corresponding integrals. The proof can be found in Appendix A.

The autocovariance functions are separable, but are not translation invariant because of the trigonometric terms depending on $\xi+\xi^{\prime}$. In addition, the autocovariance of the real and imaginary parts of $\widehat{n}$ does not vanish. Rigorously speaking, these components are thus neither stationary, nor independent. However, the Wigner-Ville transform $\mathcal{W} t_{\sigma}$ is concentrated in space around the peak of $w_{\sigma}$, and in frequency around the peak of $\widehat{w_{\sigma}}$, that is, around $(0,0)$. As a consequence, the term $\mathcal{W} w_{\sigma}\left(\left(\xi-\xi^{\prime}\right) / 2,2 \pi f\right)$ is expected to be negligible as soon as $2 \pi f$ is "large." How large $2 \pi f$ should be depends on the analysis window $w_{\boldsymbol{\sigma}}$.

The next section gives an estimation of the noise in the phase maps, and argue that the Wigner-Ville term can be actually neglected for the considered windows.

\subsection{Noise in the phase maps}

The measured phase $\widetilde{\phi_{x}}(\boldsymbol{\xi}) \in[0,2 \pi)$ writes, from (51) and $(7)$ :

$$
\widetilde{\phi_{x}}(\boldsymbol{\xi})=\arctan \left(\frac{\operatorname{Im}(\Psi(\boldsymbol{\xi}, 0))+\operatorname{Im}(\widehat{n}(\boldsymbol{\xi}))}{\operatorname{Re}(\Psi(\boldsymbol{\xi}, 0))+\operatorname{Re}(\widehat{n}(\boldsymbol{\xi}))}\right)
$$

\footnotetext{
${ }^{1}$ For any real-valued function $f$, the Wigner-Ville transform $[7,16,22]$ of $f$ is given by $\mathcal{W} f(x, \lambda)=$ $\int f(x+\tau / 2) f(x-\tau / 2) e^{-i \tau \lambda} \mathrm{d} \tau$. It is a real-valued function.
} 
Assuming that the noise variance is low with respect to $|\Psi(\boldsymbol{\xi}, 0)|^{2}$, it is possible to neglect the effect of phase jumps due to noise and to obtain an approximation of $\widetilde{\phi_{x}}$ via a Taylor expansion of arctan. Indeed, since

$$
\begin{aligned}
\arctan \left(\frac{y}{x}\right)=\arctan \left(\frac{y_{0}}{x_{0}}\right)-\frac{y_{0}}{x_{0}^{2}+y_{0}^{2}} & \left(x-x_{0}\right) \\
& +\frac{x_{0}}{x_{0}^{2}+y_{0}^{2}}\left(y-y_{0}\right)+o\left(\left\|\left(x-x_{0}, y-y_{0}\right)\right\|_{2}\right)
\end{aligned}
$$

and with $x_{0}=\operatorname{Re}(\Psi(\boldsymbol{\xi}, 0)), y_{0}=\operatorname{Im}(\Psi(\boldsymbol{\xi}, 0)), x=\operatorname{Re}(\widetilde{\Psi}(\boldsymbol{\xi}, 0))$, and $y=\operatorname{Im}(\widetilde{\Psi}(\boldsymbol{\xi}, 0))$, a first-order approximation of $\widetilde{\phi_{x}}$ is:

$$
\widetilde{\phi_{x}}(\boldsymbol{\xi}) \simeq \operatorname{angle}(\Psi(\boldsymbol{\xi}, 0))-\frac{\operatorname{Im}(\Psi(\boldsymbol{\xi}, 0))}{|\Psi(\boldsymbol{\xi}, 0)|^{2}} \operatorname{Re}(\widehat{n})(\boldsymbol{\xi})+\frac{\operatorname{Re}(\Psi(\boldsymbol{\xi}, 0))}{|\Psi(\boldsymbol{\xi}, 0)|^{2}} \operatorname{Im}(\widehat{n})(\boldsymbol{\xi})
$$

Let us define $\mathcal{R}(\boldsymbol{\xi})=\operatorname{Re}(\Psi(\boldsymbol{\xi}, 0)), \mathcal{I}(\boldsymbol{\xi})=\operatorname{Im}(\Psi(\boldsymbol{\xi}, 0)), \mathcal{A}(\boldsymbol{\xi})=|\Psi(\boldsymbol{\xi}, 0)|, \mathcal{R} \widehat{n}(\boldsymbol{\xi})=$ $\operatorname{Re}(\widehat{n}(\boldsymbol{\xi}))$, and $\mathcal{I} \widehat{n}(\boldsymbol{\xi})=\operatorname{Im}(\widehat{n}(\boldsymbol{\xi}))$. With (57), the phase becomes

$$
\widetilde{\phi_{x}}(\boldsymbol{\xi}) \simeq \operatorname{angle}(\Psi(\boldsymbol{\xi}, 0))+\widetilde{n}(\boldsymbol{\xi})
$$

where $\widetilde{n}(\boldsymbol{\xi})$ is a Gaussian random variable with mean 0 and autocovariance function :

$$
\begin{array}{r}
\operatorname{Cov}\left(\widetilde{n}(\boldsymbol{\xi}), \widetilde{n}\left(\boldsymbol{\xi}^{\prime}\right)\right)=\frac{\mathcal{I}(\boldsymbol{\xi}) \mathcal{I}\left(\boldsymbol{\xi}^{\prime}\right)}{\mathcal{A}(\boldsymbol{\xi})^{2} \mathcal{A}\left(\boldsymbol{\xi}^{\prime}\right)^{2}} \operatorname{Cov}\left(\mathcal{R} \widehat{n}(\boldsymbol{\xi}), \mathcal{R} \widehat{n}\left(\boldsymbol{\xi}^{\prime}\right)\right)+\frac{\mathcal{R}(\boldsymbol{\xi}) \mathcal{R}\left(\boldsymbol{\xi}^{\prime}\right)}{\mathcal{A}(\boldsymbol{\xi})^{2} \mathcal{A}\left(\boldsymbol{\xi}^{\prime}\right)^{2}} \operatorname{Cov}\left(\mathcal{I} \widehat{n}(\boldsymbol{\xi}), \mathcal{I} \widehat{n}\left(\boldsymbol{\xi}^{\prime}\right)\right) \\
-\left(\frac{\mathcal{I}(\boldsymbol{\xi}) \mathcal{R}\left(\boldsymbol{\xi}^{\prime}\right)}{\mathcal{A}(\boldsymbol{\xi})^{2} \mathcal{A}\left(\boldsymbol{\xi}^{\prime}\right)^{2}}+\frac{\mathcal{R}(\boldsymbol{\xi}) \mathcal{I}\left(\boldsymbol{\xi}^{\prime}\right)}{\mathcal{A}(\boldsymbol{\xi})^{2} \mathcal{A}\left(\boldsymbol{\xi}^{\prime}\right)^{2}}\right) \operatorname{Cov}\left(\mathcal{R} \widehat{n}(\boldsymbol{\xi}), \mathcal{I} \widehat{n}\left(\boldsymbol{\xi}^{\prime}\right)\right)
\end{array}
$$

Let $\psi(\boldsymbol{\xi})$ be an argument of $\Psi(\boldsymbol{\xi})$. Since

$$
\begin{gathered}
\sin (\psi(\xi, \eta)) \sin \left(\psi\left(\xi^{\prime}, \eta^{\prime}\right)\right) \cos \left(2 \pi f\left(\xi+\xi^{\prime}\right)\right)-\cos (\psi(\xi, \eta)) \cos \left(\psi\left(\xi^{\prime}, \eta^{\prime}\right)\right) \cos \left(2 \pi f\left(\xi+\xi^{\prime}\right)\right) \\
-\left(\operatorname { s i n } ( \psi ( \xi , \eta ) ) \operatorname { c o s } \left(\psi\left(\xi^{\prime}, \eta^{\prime}\right)+\cos (\psi(\xi, \eta)) \sin \left(\psi\left(\xi^{\prime}, \eta^{\prime}\right)\right) \sin \left(2 \pi f\left(\xi+\xi^{\prime}\right)\right)\right.\right. \\
=-\cos \left(2 \pi f\left(\xi+\xi^{\prime}\right)-\left(\psi(\xi, \eta)+\psi\left(\xi^{\prime}, \eta^{\prime}\right)\right)\right)
\end{gathered}
$$

and:

$$
\sin (\psi(\xi, \eta)) \sin \left(\psi\left(\xi^{\prime}, \eta^{\prime}\right)\right)+\cos (\psi(\xi, \eta)) \cos \left(\psi\left(\xi^{\prime}, \eta^{\prime}\right)\right)=\cos \left(\psi(\xi, \eta)-\psi\left(\xi^{\prime}, \eta^{\prime}\right)\right)
$$

we obtain from Proposition 1:

$$
\begin{gathered}
\operatorname{Cov}\left(\widetilde{n}(\boldsymbol{\xi}), \widetilde{n}\left(\boldsymbol{\xi}^{\prime}\right)\right)=\frac{v \Delta_{x} \Delta_{y}}{2 \mathcal{A}(\boldsymbol{\xi})^{2} \mathcal{A}\left(\boldsymbol{\xi}^{\prime}\right)^{2}} \\
\cdot\left(\cos \left(\psi(\boldsymbol{\xi})-\psi\left(\boldsymbol{\xi}^{\prime}\right)\right) w_{\sigma_{x}}^{\mathbf{x}} * w_{\sigma_{x}}^{\mathbf{x}}\left(\xi-\xi^{\prime}\right)\right. \\
\left.-\frac{1}{2} \mathcal{W} w_{\sigma_{x}}^{\mathbf{x}}\left(\left(\xi-\xi^{\prime}\right) / 2,2 \pi f\right) \cdot \cos \left(2 \pi f\left(\xi+\xi^{\prime}\right)-\left(\psi(\boldsymbol{\xi})+\psi\left(\boldsymbol{\xi}^{\prime}\right)\right)\right)\right) \\
\cdot w_{\sigma_{y}}^{\mathbf{y}} * w_{\sigma_{y}}^{\mathbf{y}}\left(\eta-\eta^{\prime}\right)
\end{gathered}
$$


Since the phase variations are locally limited, we can $\operatorname{simplify} \cos \left(\psi(\xi, \eta)-\psi\left(\xi^{\prime}, \eta^{\prime}\right)\right)$ to 1. Moreover, it is proved in [32] that $\mathcal{A}(\boldsymbol{\xi})=|\Psi(\xi, \eta, 0)|$ is practically constant, equal to $K=\left|d_{1}\right| \gamma A / 2$. (Note that this also justifies the Taylor expansion of arctan to obtain (56), since practically $v<<K^{2}$.)

The covariance of the noise thus simplifies into

$$
\begin{aligned}
\operatorname{Cov}\left(\widetilde{n}(\boldsymbol{\xi}), \widetilde{n}\left(\boldsymbol{\xi}^{\prime}\right)\right)=\frac{v \Delta_{x} \Delta_{y}}{2 K^{2}}\left(w_{\sigma_{x}}^{\mathbf{x}} * w_{\sigma_{x}}^{\mathbf{x}}\left(\xi-\xi^{\prime}\right)\right. \\
\left.-\frac{1}{2} \mathcal{W} w_{\sigma_{x}}^{\mathbf{x}}\left(\left(\xi-\xi^{\prime}\right) / 2,2 \pi f\right) \cdot \cos \left(2 \pi f\left(\xi+\xi^{\prime}\right)-\left(\psi(\xi, \eta)+\psi\left(\xi^{\prime}, \eta^{\prime}\right)\right)\right)\right) \\
\cdot w_{\sigma_{y}}^{\mathbf{y}} * w_{\sigma_{y}}^{\mathbf{y}}\left(\eta-\eta^{\prime}\right)
\end{aligned}
$$

We can see that the covariance of $\widetilde{n}$ is not invariant to translations, because of the cosine term. In other terms, the noise $\widetilde{n}$ on the phase map is not stationary. However, it turns out that the term depending on the Wigner-Ville transform cancels out in practice. The Wigner-Ville transform of the considered windows can be found in Table 2. Let us write $\alpha=\frac{\left|\xi-\xi^{\prime}\right|}{2}$. Figure 9 shows, for the considered window functions, the plots of the ratio $\mathcal{W} w_{\sigma}(\alpha, 2 \pi f) / \mathcal{W} w_{\sigma}(0,0)$ as a function of $\alpha f$, for several values of $\sigma f$ (satisfying the conditions on the window size established in Section 2.2 for the rectangular and triangular windows). We can see that this quantity is always smaller than a few percent for the triangular window, and for the rectangular window as soon as af $\geq 2$. It is always numerically negligible for the Gaussian window. Since $w_{\sigma} * w_{\sigma}(0)=\left\|w_{\sigma}\right\|_{2}^{2}=$ $\frac{1}{2} \mathcal{W} w_{\sigma}(0,0)$, this means that the correlation function of $\widetilde{n}$ can be practically written as

$$
\operatorname{Corr}\left(\widetilde{n}(\boldsymbol{\xi}), \widetilde{n}\left(\boldsymbol{\xi}^{\prime}\right)\right)=\frac{v \Delta_{x} \Delta_{y}}{\left.2 K^{2}\left\|w_{\sigma_{x}}^{\mathbf{x}}\right\|\right|_{2} ^{2}\left\|w_{\sigma_{y}}^{\mathbf{y}}\right\|_{2}^{2}} \cdot w_{\sigma_{x}}^{\mathbf{x}} * w_{\sigma_{x}}^{\mathbf{x}}\left(\xi-\xi^{\prime}\right) \cdot w_{\sigma_{y}}^{\mathbf{y}} * w_{\sigma_{y}}^{\mathbf{y}}\left(\eta-\eta^{\prime}\right)
$$

or the covariance as

$$
\operatorname{Cov}\left(\widetilde{n}(\boldsymbol{\xi}), \widetilde{n}\left(\boldsymbol{\xi}^{\prime}\right)\right)=\frac{v \Delta_{x} \Delta_{y}}{2 K^{2}} w_{\sigma_{x}}^{\mathbf{x}} * w_{\sigma_{x}}^{\mathbf{x}}\left(\xi-\xi^{\prime}\right) w_{\sigma_{y}}^{\mathbf{y}} * w_{\sigma_{y}}^{\mathbf{y}}\left(\eta-\eta^{\prime}\right)
$$

In particular, the variance of the noise on the phase is:

$$
\operatorname{Var}(\widetilde{n})=\frac{v \Delta_{x} \Delta_{y}}{2 K^{2}}\left\|w_{\sigma_{x}}^{\mathbf{x}}\right\|_{2}^{2}\left\|w_{\sigma_{y}}^{\mathbf{y}}\right\|_{2}^{2}
$$

In these equations, the convolution of the window by itself can be obtained via the Wigner-Ville transform with the following relation:

$$
w_{\sigma} * w_{\sigma}(t)=\frac{1}{2} \mathcal{W} w_{\sigma}(t / 2,0)
$$




\begin{tabular}{|c||c|}
\hline Window & Wigner-Ville transform \\
\hline \hline Rectangular & $\mathcal{W} r_{a}(x, \lambda)=\frac{1}{2 \lambda a^{2}} \sin (2(a-|x|) \lambda) \mathbb{1}_{[-a, a]}(x)$ \\
$r_{a}(x)=\frac{1}{2 a} \mathbb{1}_{[-a, a]}(x)$ & $\mathcal{W} r_{a}(x, 0)=\frac{a-|x|}{a^{2}} \mathbb{1}_{[-a, a]}(x)$ \\
\hline $\begin{array}{c}\text { Triangular } \\
t_{b}(x)=\frac{b-|x|}{b^{2}} \mathbb{1}_{[-b, b]}(x)\end{array}$ & $\begin{array}{r}\mathcal{W} t_{b}(x, \lambda)=\frac{2}{b^{4} \lambda^{2}}\left[\left(-(b-|x|) \cos (2 \lambda(b-|x|))+\frac{1}{2 \lambda} \sin (2 \lambda(b-|x|))\right) \mathbb{1}_{[-b, b]}(x)\right. \\
\mathcal{W} t_{b}(x, 0)=\frac{8(b-|x|)^{3}}{3 b^{4}} \mathbb{1}_{[-b, b]}(x)-\frac{4(b-2|x|)^{3}}{3 b^{4}} \mathbb{1}_{[-b / 2, b / 2]}(x)\end{array}$ \\
\hline$g_{\sigma}(x)=\frac{1}{\sqrt{2 \pi} \sigma} e^{-x^{2} /\left(2 \sigma^{2}\right)}$ & $\mathcal{W} g_{\sigma}(x, \lambda)=\frac{1}{\sqrt{\pi} \sigma} e^{-x^{2} / \sigma^{2}-\sigma^{2} \lambda^{2}}$ \\
\hline
\end{tabular}

Table 2: Wigner-Ville transforms of the rectangular, triangular, and Gaussian windows. See Appendix B.

\subsection{Noise in the phase derivative maps}

Characterizing the noise in the derivative maps in addition to the noise in the phase maps themselves is of prime importance in the current context. The reason is that the mechanical response of the materials or structures, which are expected to be studied with such contactless measuring systems, directly depends on the strain components through the constitutive equations, and not on the displacement directly. Strains being derived from the displacement by differentiation (see (5)), and displacement being proportional to phase changes between reference and current images of the regularly marked deformed surface (see 4), noise in the phase derivative maps must also be carefully studied.

Let $\frac{\partial \widetilde{n}}{\partial \xi}$ and $\frac{\partial \widetilde{n}}{\partial \eta}$ be the noise on the phase derivatives. Since these derivatives are obtained with finite difference schemes, we identify these random fields with the meansquare partial derivatives of the stationary random field $\widetilde{n}[1]$. The covariance of these random fields is the opposite of the second derivative of the covariance of $\widetilde{n}$, as soon as these second derivatives exist and are finite (see Corollary 2.4.1 in [1]). In this case,

$$
\operatorname{Cov}\left(\frac{\partial \widetilde{n}}{\partial \xi}(\boldsymbol{\xi}), \frac{\partial \widetilde{n}}{\partial \xi}\left(\boldsymbol{\xi}^{\prime}\right)\right)=-\frac{v \Delta_{x} \Delta_{y}}{2 K^{2}} \cdot\left(w_{\sigma_{x}}^{\mathbf{x}} * w_{\sigma_{x}}^{\mathbf{x}}\right)^{\prime \prime}\left(\xi-\xi^{\prime}\right) w_{\sigma_{y}}^{\mathbf{y}} * w_{\sigma_{y}}^{\mathbf{y}}\left(\eta-\eta^{\prime}\right)
$$

and:

$$
\operatorname{Cov}\left(\frac{\partial \widetilde{n}}{\partial \xi}(\boldsymbol{\xi}), \frac{\partial \widetilde{n}}{\partial \xi}\left(\boldsymbol{\xi}^{\prime}\right)\right)=-\frac{v \Delta_{x} \Delta_{y}}{2 K^{2}} \cdot w_{\sigma_{x}}^{\mathbf{x}} * w_{\sigma_{x}}^{\mathbf{x}}\left(\xi-\xi^{\prime}\right)\left(w_{\sigma_{y}}^{\mathbf{y}} * w_{\sigma_{y}}^{\mathbf{y}}\right)^{\prime \prime}\left(\eta-\eta^{\prime}\right)
$$

where $f^{\prime \prime}$ denotes the second derivatives of any real-valued function $f$. 

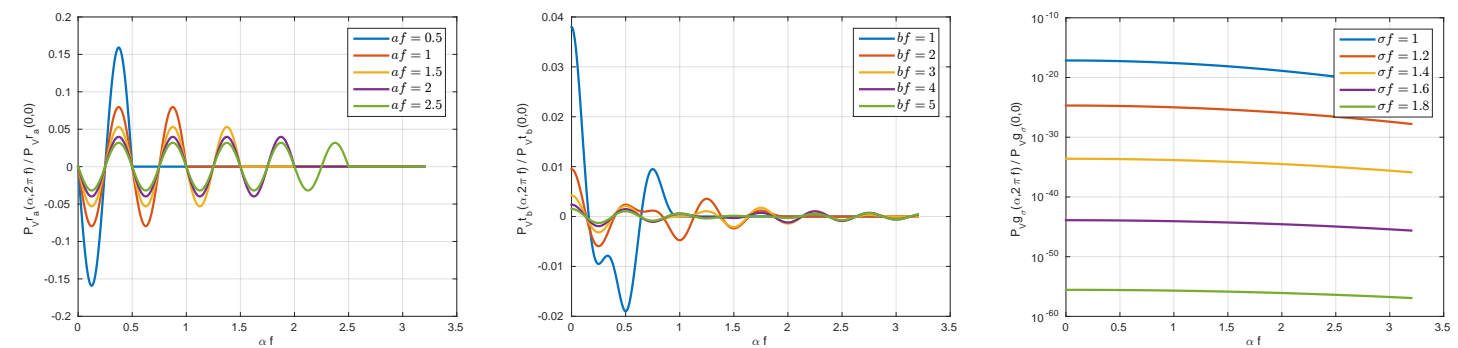

Figure 9: Plot of $\mathcal{W} w_{\sigma}(\alpha, 2 \pi f) / \mathcal{W} w_{\sigma}(0,0)$ as a function of $\alpha f$, for several values of $\sigma f$. From left to right: rectangular window $\left(w_{\sigma}=r_{a}\right)$, triangular window $\left(w_{\sigma}=t_{b}\right)$, Gaussian window $\left(w_{\sigma}=g_{\sigma}\right.$, logarithmic scale on the ordinate).

In particular, the variance on the phase derivatives writes:

$$
\begin{aligned}
\operatorname{Var}\left(\frac{\partial \widetilde{n}}{\partial \xi}(\xi, \eta)\right) & =-\frac{v \Delta_{x} \Delta_{y}}{2 K^{2}}\left\|w_{\sigma_{y}}^{\mathbf{y}}\right\|_{2}^{2}\left(w_{\sigma_{x}}^{\mathbf{x}} * w_{\sigma_{x}}^{\mathbf{x}}\right)^{\prime \prime}(0) \\
\operatorname{Var}\left(\frac{\partial \widetilde{n}}{\partial \eta}(\xi, \eta)\right) & =-\frac{v \Delta_{x} \Delta_{y}}{2 K^{2}}\left\|w_{\sigma_{x}}^{\mathbf{x}}\right\|_{2}^{2}\left(w_{\sigma_{y}}^{\mathbf{y}} * w_{\sigma_{y}}^{\mathbf{y}}\right)^{\prime \prime}(0)
\end{aligned}
$$

\subsection{Noise variance and covariance for the rectangular, triangular, and Gaussian windows}

With $\left(w_{\sigma} * w_{\sigma}\right)^{\prime \prime}=\frac{1}{2} \mathcal{W} w_{\sigma}(\dot{\overline{2}}, 0)^{\prime \prime}$ and the results gathered in Table 2, we obtain the following expressions:

- For the rectangular window, $\mathcal{W} w_{\sigma}$ is only differentiable in the sense of distributions, yielding

$$
\left(r_{a} * r_{a}\right)^{\prime \prime}(u)=\frac{1}{4 a^{2}}\left(\delta_{-2 a}(u)-2 \delta_{0}(u)+\delta_{2 a}(u)\right)
$$

This does not satisfy the hypothesis of the theorem on the differentiability of random fields. It is thus not possible to use the continuous approach. Instead, we have to consider the finite difference scheme which is used. The calculation is detailed in Appendix C.

- For the triangular window:

$$
\left(t_{b} * t_{b}\right)^{\prime \prime}(u)=\frac{2}{b^{4}}\left((b-|u| / 2) \mathbb{1}_{[-2 b, 2 b]}(u)-2(b-|u|) \mathbb{1}_{[-b, b]}(u)\right)
$$

- For the Gaussian window:

$$
\left(g_{\sigma} * g_{\sigma}\right)^{\prime \prime}(u)=-\frac{1}{4 \sqrt{\pi} \sigma^{3}}\left(1-\frac{u^{2}}{2 \sigma^{2}}\right) e^{-u^{2} /\left(4 \sigma^{2}\right)}
$$


Plugging these results (and the result of Appendix $\mathrm{C}$ for the rectangular profile) in (65), (68), and (69), we obtain the expressions for each of the windows of interest.

In the following, we use

$$
\widetilde{\delta}_{\alpha}(t)=\left(1-\frac{|t-\alpha|}{2 \Delta_{x}}\right) \mathbb{1}_{\left[\alpha-2 \Delta_{x}, \alpha+2 \Delta_{x}\right]}(t)
$$

and the covariance function of the stationary random variables:

$$
\left\{\begin{array}{l}
C(s, t)=\operatorname{Cov}\left(\widetilde{n}(\boldsymbol{\xi}), \widetilde{n}\left(\boldsymbol{\xi}^{\prime}\right)\right) \\
C_{\xi}(s, t)=\operatorname{Cov}\left(\frac{\partial \widetilde{n}}{\partial \xi}(\boldsymbol{\xi}), \frac{\partial \widetilde{n}}{\partial \xi}\left(\boldsymbol{\xi}^{\prime}\right)\right) \\
C_{\eta}(s, t)=\operatorname{Cov}\left(\frac{\partial \widetilde{n}}{\partial \eta}(\boldsymbol{\xi}), \frac{\partial \widetilde{n}}{\partial \eta}\left(\boldsymbol{\xi}^{\prime}\right)\right)
\end{array}\right.
$$

with $(s, t)=\left(\xi-\xi^{\prime}, \eta-\eta^{\prime}\right)$.

\subsubsection{Birectangular window}

$$
\begin{aligned}
C(s, t) & =\frac{v \Delta_{x} \Delta_{y}}{8 K^{2} a^{2}}(1-|s| /(2 a))(1-|t| /(2 a)) \mathbb{1}_{[-2 a, 2 a] \times[-2 a, 2 a]}(s, t) \\
C_{\xi}(s, t) & =\frac{v \Delta_{x} \Delta_{y}}{32 K^{2} a^{3} \Delta_{x}}(1-|t| /(2 a))\left(-\widetilde{\delta}_{-2 a}+2 \widetilde{\delta}_{0}-\widetilde{\delta}_{2 a}\right)(s) \mathbb{1}_{[-2 a, 2 a]}(t) \\
C_{\eta}(s, t) & =\frac{v \Delta_{x} \Delta_{y}}{32 K^{2} a^{3} \Delta_{x}}(1-|s| /(2 a))\left(-\widetilde{\delta}_{-2 a}+2 \widetilde{\delta}_{0}-\widetilde{\delta}_{2 a}\right)(t) \mathbb{1}_{[-2 a, 2 a]}(s)
\end{aligned}
$$

The variance of the noise on the phase is:

$$
\operatorname{Var}(\widetilde{n}(\boldsymbol{\xi}))=\frac{v \Delta_{x} \Delta_{y}}{8 K^{2} a^{2}}
$$

The variance of the noise on the phase derivative is:

$$
\operatorname{Var}\left(\frac{\partial \widetilde{n}}{\partial \xi}(\boldsymbol{\xi})\right)=\operatorname{Var}\left(\frac{\partial \widetilde{n}}{\partial \eta}(\boldsymbol{\xi})\right)=\frac{v \Delta_{x} \Delta_{y}}{16 K^{2} a^{3} \Delta_{x}}
$$

\subsubsection{Triangular-rectangular window}

$$
\begin{aligned}
& C(s, t)= \frac{v \Delta_{x} \Delta_{y}}{6 K^{2} a b}(1-|t| /(2 a)) \mathbb{1}_{[-2 a, 2 a]}(t) \\
&\left(2(1-|s| /(2 b))^{3} \mathbb{1}_{[-2 b, 2 b]}(s)-(1-|s| / b)^{3} \mathbb{1}_{[-b, b]}(s)\right) \\
& C_{\xi}(s, t)=\frac{v \Delta_{x} \Delta_{y}}{2 K^{2} a b^{3}}(1-|t| /(2 a)) \mathbb{1}_{[-2 a, 2 a]}(t) \\
&\left(2(1-|s| / b) \mathbb{1}_{[-b, b]}(s)-(1-|s| /(2 b)) \mathbb{1}_{[-2 b, 2 b]}(s)\right)
\end{aligned}
$$




$$
\begin{aligned}
C_{\eta}(s, t)=\frac{v \Delta_{x} \Delta_{y}}{24 K^{2} a^{2} b \Delta_{x}}\left(-\widetilde{\delta}_{-2 a}+2 \widetilde{\delta}_{0}-\widetilde{\delta}_{2 a}\right)(t) \\
\quad\left(2\left(1-\left|\xi-\xi^{\prime}\right| /(2 b)\right)^{3} \mathbb{1}_{[-2 b, 2 b]}(s)-(1-|s| / b)^{3} \mathbb{1}_{[-b, b]}(s)\right)
\end{aligned}
$$

The variance of the noise on the phase is:

$$
\operatorname{Var}(\widetilde{n}(\boldsymbol{\xi}))=\frac{v \Delta_{x} \Delta_{y}}{6 K^{2} a b}
$$

The variance of the noise on the phase derivative is:

$$
\begin{gathered}
\operatorname{Var}\left(\frac{\partial \widetilde{n}}{\partial \xi}(\boldsymbol{\xi})\right)=\frac{v \Delta_{x} \Delta_{y}}{2 K^{2} a b^{3}} \\
\operatorname{Var}\left(\frac{\partial \widetilde{n}}{\partial \eta}(\boldsymbol{\xi})\right)=\frac{v \Delta_{x} \Delta_{y}}{12 K^{2} a^{2} b \Delta_{x}}
\end{gathered}
$$

\subsubsection{Bitriangular window}

$$
\begin{gathered}
C(s, t)=\frac{2 v \Delta_{x} \Delta_{y}}{9 K^{2} b^{2}} \cdot\left(2(1-|s| /(2 b))^{3} \mathbb{1}_{[-2 b, 2 b]}(s)-(1-|s| / b)^{3} \mathbb{1}_{[-b, b]}(s)\right) \\
\cdot\left(2(1-|t| /(2 b))^{3} \mathbb{1}_{[-2 b, 2 b]}(t)-(1-|t| / b)^{3} \mathbb{1}_{[-b, b]}(t)\right) \\
C_{\xi}(s, t)=\frac{2 v \Delta_{x} \Delta_{y}}{3 K^{2} b^{4}} \cdot\left(2(1-|s| / b) \mathbb{1}_{[-b, b]}(s)-(1-|s| /(2 b)) \mathbb{1}_{[-2 b, 2 b]}(s)\right) \\
\cdot\left(2(1-|t| /(2 b))^{3} \mathbb{1}_{[-2 b, 2 b]}(t)-(1-|t| / b)^{3} \mathbb{1}_{[-b, b]}(t)\right) \\
C_{\eta}(s, t)=\frac{2 v \Delta_{x} \Delta_{y}}{3 K^{2} b^{4}} \\
\cdot\left(2(1-|t| / b) \mathbb{1}_{[-b, b]}(t)-(1-|t| /(2 b)) \mathbb{1}_{[-2 b, 2 b]}(t)\right) \\
\cdot\left(2(1-|s| /(2 b))^{3} \mathbb{1}_{[-2 b, 2 b]}(s)-(1-|s| / b)^{3} \mathbb{1}_{[-b, b]}(s)\right)
\end{gathered}
$$

The variance of the noise on the phase is:

$$
\operatorname{Var}(\widetilde{n}(\boldsymbol{\xi}))=\frac{2 v \Delta_{x} \Delta_{y}}{9 K^{2} b^{2}}
$$

The variance of the noise on the phase derivative is:

$$
\operatorname{Var}\left(\frac{\partial \widetilde{n}}{\partial \xi}(\boldsymbol{\xi})\right)=\operatorname{Var}\left(\frac{\partial \widetilde{n}}{\partial \eta}(\boldsymbol{\xi})\right)=\frac{2 v \Delta_{x} \Delta_{y}}{3 K^{2} b^{4}}
$$




\subsubsection{Gaussian window}

$$
\begin{gathered}
C(s, t)=\frac{v \Delta_{x} \Delta_{y}}{8 K^{2} \pi \sigma^{2}} e^{-s^{2} /\left(4 \sigma^{2}\right)-t^{2} /\left(4 \sigma^{2}\right)} \\
C_{\xi}(s, t)=\frac{v \Delta_{x} \Delta_{y}}{16 \pi K^{2} \sigma^{4}}\left(1-\frac{s^{2}}{2 \sigma^{2}}\right) e^{-s^{2} /\left(2 \sigma^{2}\right)-t^{2} /\left(2 \sigma^{2}\right)} \\
C_{\eta}(s, t)=\frac{v \Delta_{x} \Delta_{y}}{16 \pi K^{2} \sigma^{4}}\left(1-\frac{t^{2}}{2 \sigma^{2}}\right) e^{-s^{2} /\left(4 \sigma^{2}\right)-t^{2} /\left(4 \sigma^{2}\right)}
\end{gathered}
$$

The variance of the noise on the phase is:

$$
\operatorname{Var}(\widetilde{n}(\boldsymbol{\xi}))=\frac{v \Delta_{x} \Delta_{y}}{8 K^{2} \pi \sigma^{2}}
$$

The variance of the noise on the phase derivative is:

$$
\operatorname{Var}\left(\frac{\partial \widetilde{n}}{\partial \xi}(\boldsymbol{\xi})\right)=\operatorname{Var}\left(\frac{\partial \widetilde{n}}{\partial \eta}(\boldsymbol{\xi})\right)=\frac{v \Delta_{x} \Delta_{y}}{16 \pi K^{2} \sigma^{4}}
$$

In this particular case, we obtain the same values as the ones obtained in [32] (Sections 3.2.1 and 3.2.2) by another method.

\subsection{Comparison for the same spatial resolution}

Since the measurement resolution can be expressed as the standard deviation of the noise term, we are interested in comparing the noise in the measurement maps obtained with these windows. As explained earlier in the paper, it is not obvious to compare the results obtained for different windows because the measurement resolution and the spatial resolution are linked. In order to compare the noise level in phase and phase derivative maps, we adopt the same approach as in Section 2.4, that is, we compute the noise variance for the same spatial resolution.

As in Section 2.4, this means that we express the parameters of the birectangular, triangular-rectangular, and bitriangular windows proportionally to the parameter $\sigma$ of the Gaussian window. More explicitly, we set $a=1.7137 \sigma$ for the birectangular window, $a=b=2.0434 \sigma$ for the triangular-rectangular window, and $b=2.4366 \sigma$ for the bitriangular window.

Let $v_{r}, v_{t r}, v_{t}$, and $v_{g}$ be the noise variance of the phase maps obtained with the birectangular, triangular-rectangular, bitriangular, and Gaussian windows, corresponding to (80), (85), (91), and (96), respectively.

A simple calculation gives:

- $v_{r} / v_{g}=\pi \sigma^{2} / a^{2}$, thus, $v_{r}=1.070 v_{g}$;

- $v_{t r} / v_{g}=4 \pi \sigma^{2} /(3 a b)$, thus, $v_{t r}=1.003 v_{g}$;

- $v_{t} / v_{g}=16 \pi \sigma^{2} /\left(9 b^{2}\right)$, thus, $v_{t}=0.9407 v_{g}$. 
Quite surprisingly, for a given spatial resolution (but still depending on an arbitrary $\lambda^{*}$ value), the measurement resolution is nearly the same for all the windows considered here.

Concerning the phase derivatives, $\left(v_{r, \xi}, v_{r, \eta}\right),\left(v_{t r, \xi}, v_{t r, \eta}\right),\left(v_{t, \xi}, v_{t, \eta}\right)$, and $\left(v_{g, \xi}, v_{g, \eta}\right)$ denote the noise variance of the phase derivative maps obtained with the birectangular, triangular-rectangular, bitriangular, and Gaussian windows, corresponding to (81), (8687), (92), and (97), respectively.

We obtain:

- $v_{r, \xi} / v_{g, \xi}=v_{r, \eta} / v_{g, \eta}=\pi \sigma^{4} /\left(a^{3} \Delta_{x}\right)$, thus, $v_{r, \xi}=v_{r, \eta}=0.624 \sigma / \Delta_{x} v_{g, \eta}$.

- $v_{t r, \xi} / v_{g, \xi}=8 \pi \sigma^{4} /\left(a b^{3}\right)$ and

$v_{t r, \eta} / v_{g, \eta}=2 \pi \sigma^{4} /\left(3 a^{2} b \Delta_{x}\right)$, thus $v_{t r, \xi}=1.442 v_{g, \xi}$ and $v_{t r, \eta}=0.245\left(\sigma / \Delta_{x}\right) v_{g, \eta}$.

- $v_{t, \xi} / v_{g, \xi}=v_{t, \eta} / v_{g, \eta}=32 \pi \sigma^{4} /\left(3 b^{4}\right)$, thus, $v_{t, \xi}=0.951 v_{g, \xi}$.

Since $\sigma=5$ pixels is a typical value, we can see that the noise variance (thus, the squared measurement resolution) on the phase derivative is much higher for the birectangular and triangular-rectangular windows, the bitriangular and Gaussian windows giving similar values.

\subsection{Numerical assessment}

The goal of this section is to verify that, in spite of the necessary simplifying assumptions, the covariance functions given in Section 3.4 are correct.

We generate synthetic grid images within the same framework as in Section 2.3, to which we add a Gaussian white noise of standard deviation 30 gray levels, the gray-level depth being 12 bits (such a value proved to be a realistic noise level for a real 12-bit camera such as the Sensicam QE camera used in the experiment discussed below). We compute the empirical covariance function for the noise on the phase and on its derivatives from 5,000 noisy images independently generated in this way, for the birectangular, triangular-rectangular, and bitriangular windows. Here, $a=b=12$ and $\sigma=6$.

Figure 10 shows the results for the birectangular window. In the covariance matrix we can see a residual periodic oscillation in the empirical covariance which comes from the cosine term described in Section 3.2. Although the corresponding Wigner-Ville term is small enough to neglect its effect in the phase (the empirical covariance function does not show any periodic fluctuation), it is not small enough to discard the effect of its derivatives in the phase derivative maps.

Figure 11 concerns the triangular-rectangular window. Oscillatory patterns can also be seen, once again because of the rectangular profile.

This phenomenon is no more noticeable in Figure 12 for the bitriangular window. The covariances for the Gaussian windows are shown in Figure 13. They have been already numerically assessed in [32]. We can conclude that in any cases, the empirical covariance functions are in good agreement with the theoretical formula.

Figure 14 shows the retrieved strain components in an experiment involving an openhole specimen subjected to a tensile test. Strain map estimations with each one of the 

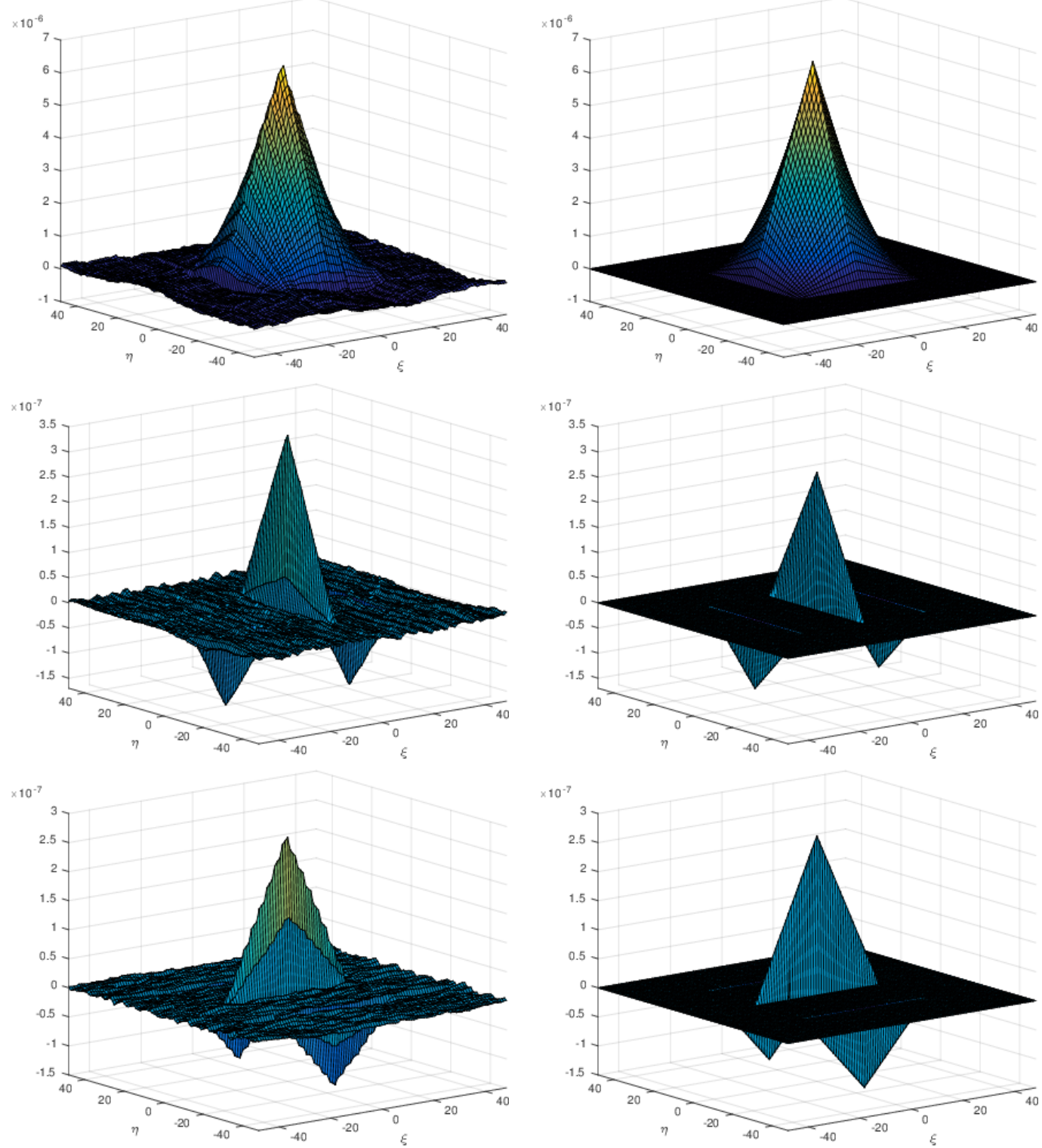

Figure 10: Birectangular window. From top to bottom: covariance function of the noise on $\phi_{x}$, covariance function of the noise on $\partial \phi_{x} / \partial \xi$, and covariance function of the noise on $\partial \phi_{x} / \partial \eta$. Left: empirical covariance. Right: theoretical covariance, cf. (77-79). 

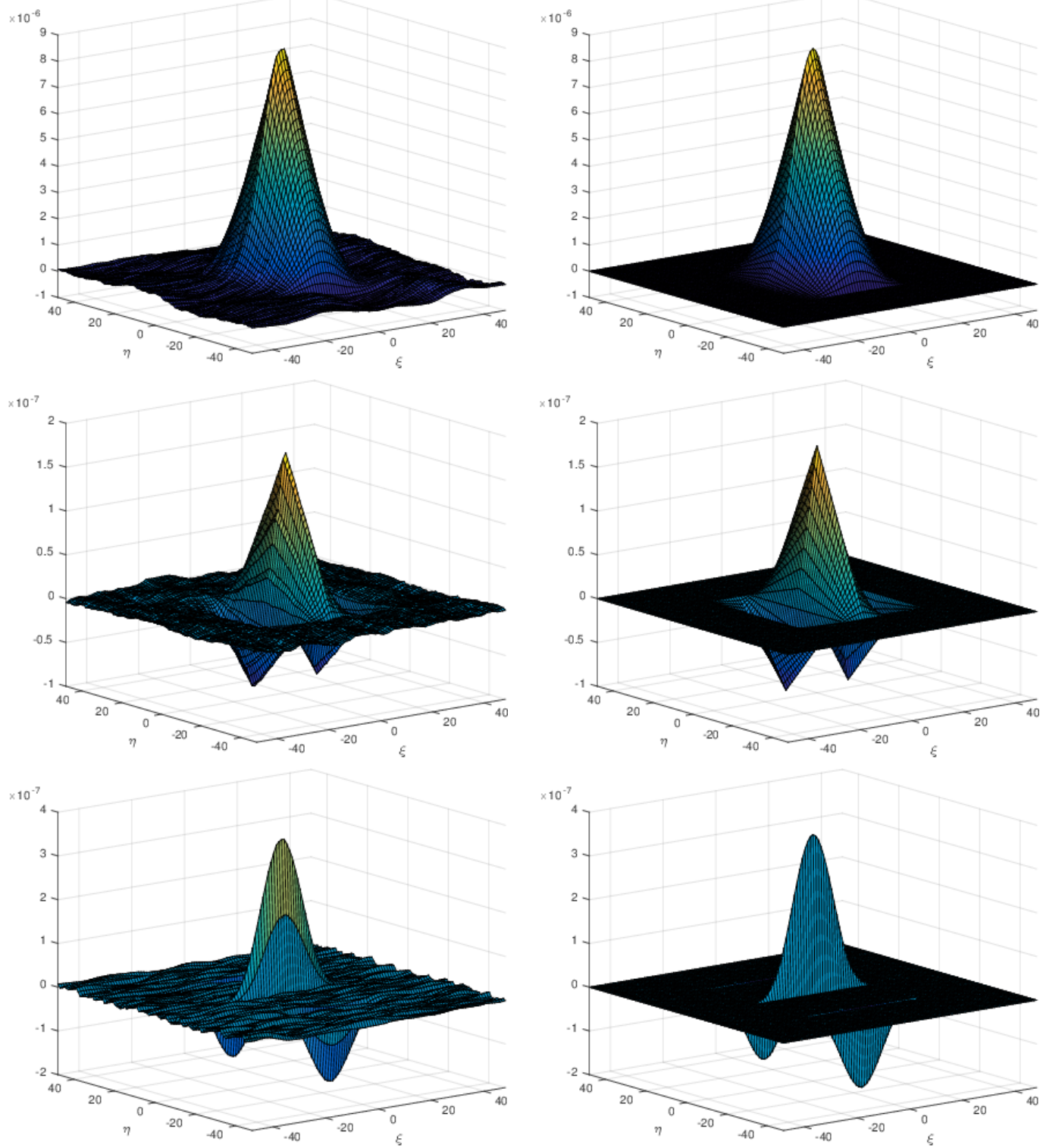

Figure 11: Triangular-rectangular window. From top to bottom: covariance function of the noise on $\phi_{x}$, covariance function of the noise on $\partial \phi_{x} / \partial \xi$, and covariance function of the noise on $\partial \phi_{x} / \partial \eta$. Left: empirical covariance. Right: theoretical covariance, cf. (82$84)$. 

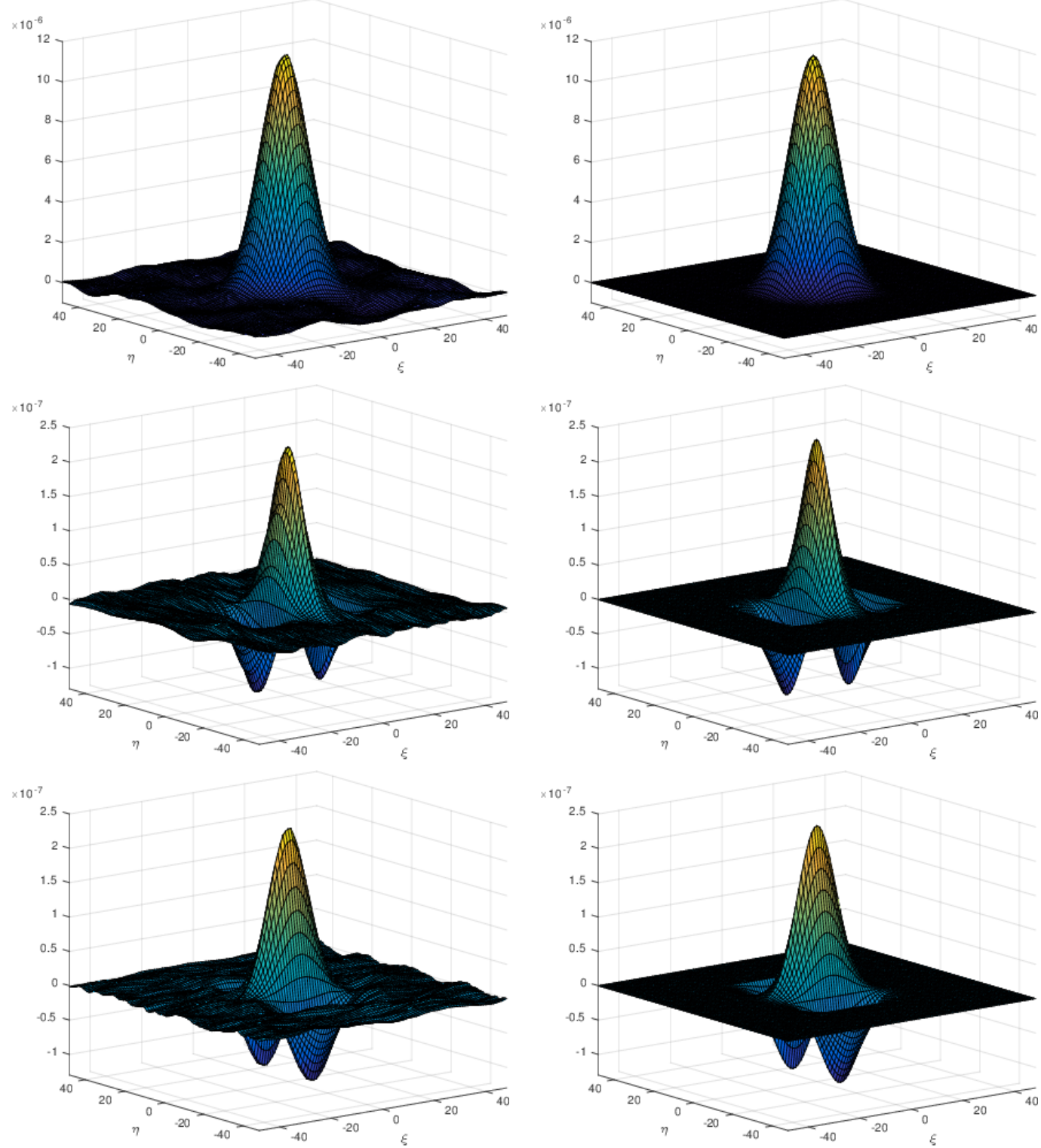

Figure 12: Bitriangular window. From top to bottom: covariance function of the noise on $\phi_{x}$, covariance function of the noise on $\partial \phi_{x} / \partial \xi$, and covariance function of the noise on $\partial \phi_{x} / \partial \eta$. Left: empirical covariance. Right: theoretical covariance, cf. (88-90). 

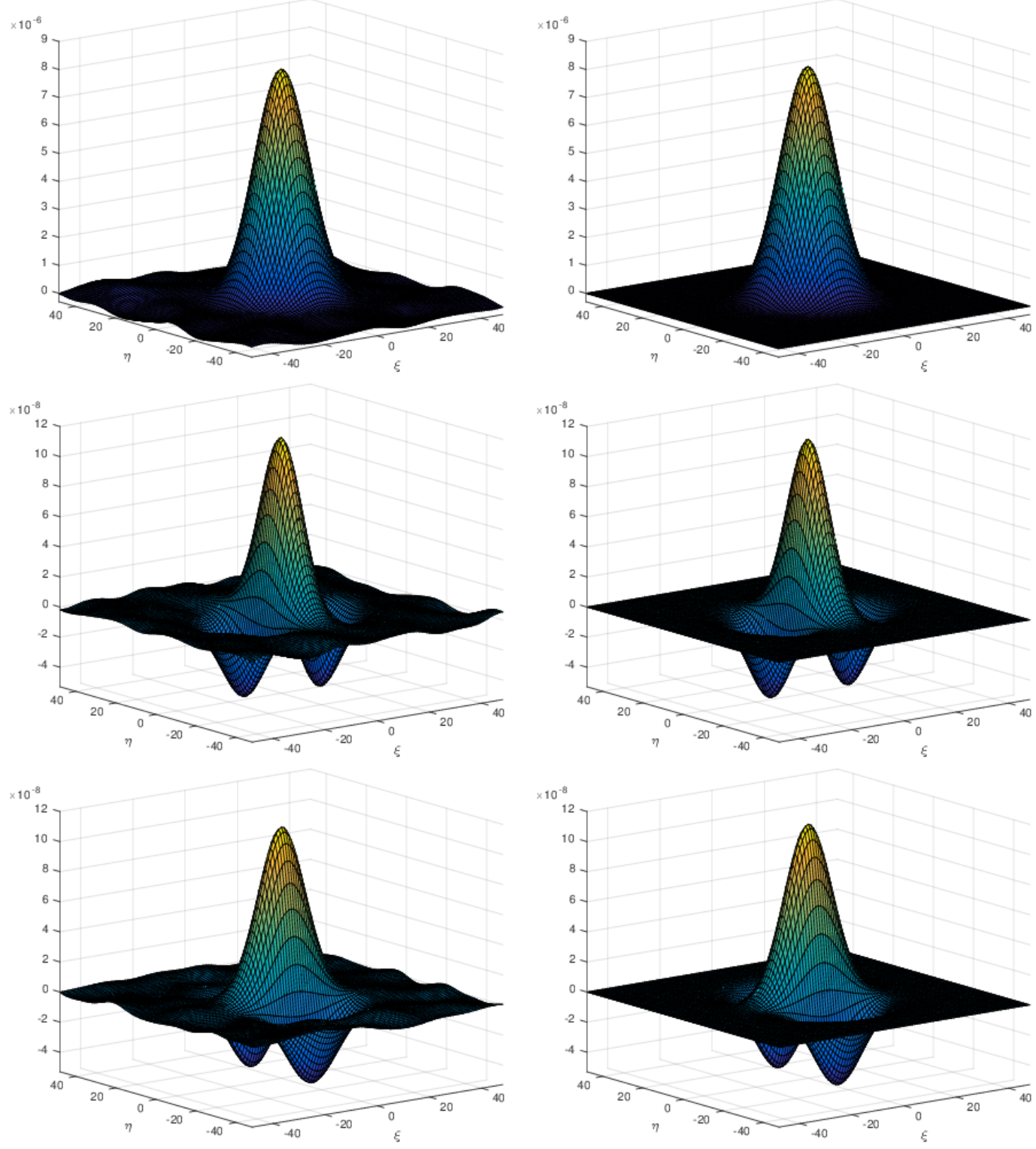

Figure 13: Gaussian window. From top to bottom: covariance function of the noise on $\phi_{x}$, covariance function of the noise on $\partial \phi_{x} / \partial \xi$, and covariance function of the noise on $\partial \phi_{x} / \partial \eta$. Left: empirical covariance. Right: theoretical covariance, cf. (93-95). 
windows studied in the present article are presented. Note that the circular pattern in the middle of the figures corresponds to the hole drilled through the specimen, the strain values have no meaning in this region. Noise manifests itself as spurious "blobs" in the strain maps. The shape of the blobs seen in the maps is directly governed by the covariance (or correlation) functions established above. The smooth covariance function given by the Gaussian window presents the advantage to give smooth blobs, instead of sharp patterns in the other cases.
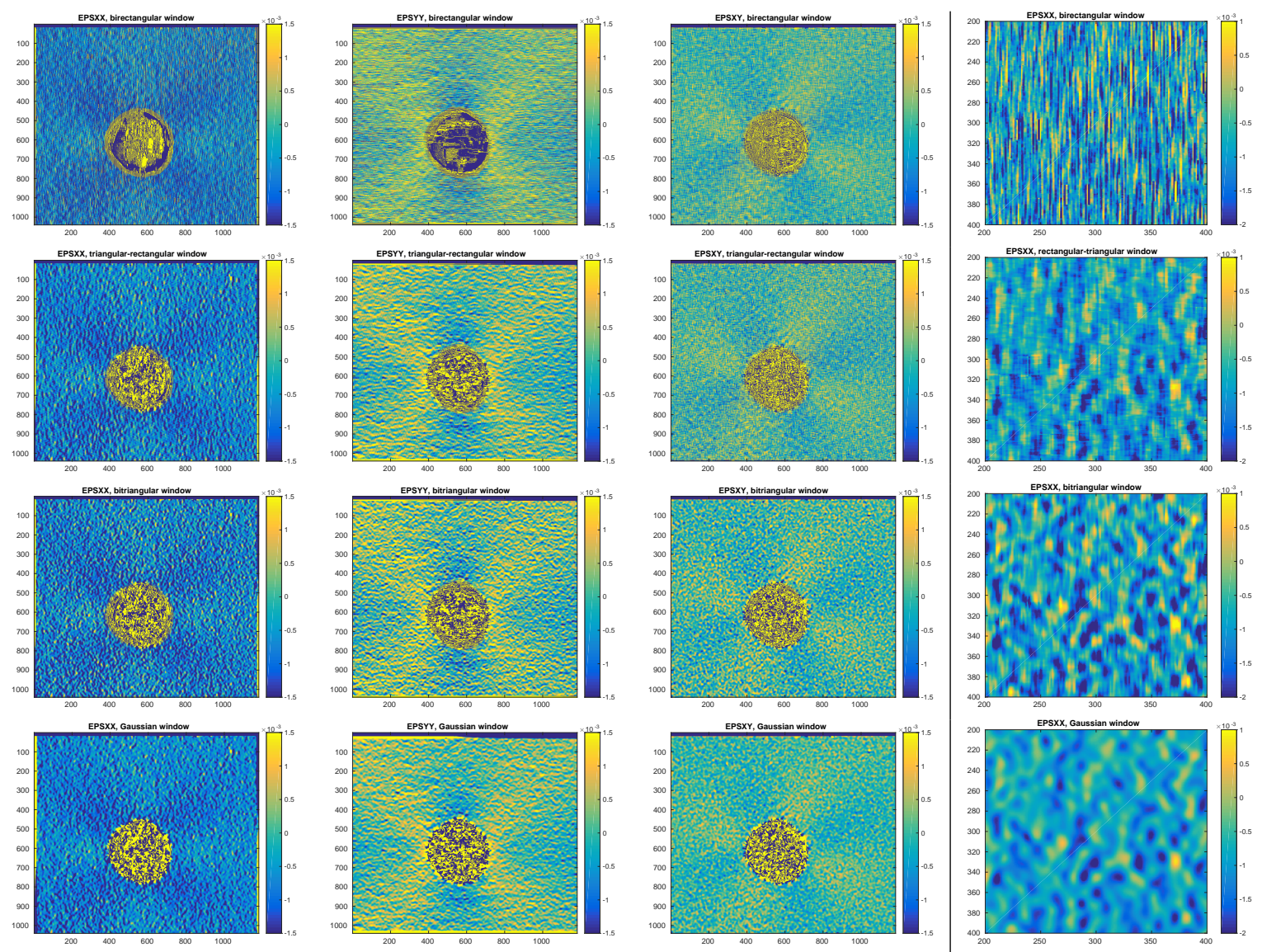

Figure 14: From left to right: strain components $\varepsilon_{x x}, \varepsilon_{y y}, \varepsilon_{x y}$, and close-up on $\varepsilon_{x x}$. From top to bottom: birectangular $(a=10)$, triangular-rectangular $(a=b=10)$, bitriangular $(b=10)$, and Gaussian window $(\sigma=5)$. 


\begin{tabular}{|c||c||c||c|c|c|c|}
\hline $\begin{array}{c}\text { Analysis } \\
\text { window }\end{array}$ & $\begin{array}{c}\text { harmonics } \\
\text { attenuation }\end{array}$ & $\begin{array}{c}\text { minimum } \\
\text { half-width }\end{array}$ & direction & $\begin{array}{c}\text { measurement } \\
\text { bias } \\
\text { Sec. } 2.4\end{array}$ & $\begin{array}{c}\text { spatial } \\
\text { resolution } \\
\text { Sec. } 2.4\end{array}$ & $\begin{array}{c}\text { measurement } \\
\text { resolution } \\
\text { Sec. 3.4 }\end{array}$ \\
\hline \hline $\begin{array}{c}\text { Birectangular } \\
r_{a}(x) r_{a}(y)\end{array}$ & Sec. 2.2 .1 & $a=p / 2$ & $x$ or $y$ & $\lambda\left(f^{\prime}\right)=1-\left|\operatorname{sinc}\left(2 a f^{\prime}\right)\right|$ & $d=a / 0.1252$ & $\frac{\sqrt{v \Delta_{x} \Delta_{y}}}{2 \sqrt{2} K a} / \frac{\sqrt{v \Delta_{x} \Delta_{y}}}{4 K a^{3 / 2} \sqrt{\Delta_{x}}}$ \\
\hline $\begin{array}{c}\text { Triangular- } \\
\text { rectangular }\end{array}$ & Sec. 2.2 .2 & $b=p$ & $x$ & $\lambda\left(f^{\prime}\right)=1-\operatorname{sinc}^{2}\left(b f^{\prime}\right)$ & $d=a / 0.1780$ & $\frac{\sqrt{v \Delta_{x} \Delta_{y}}}{K \sqrt{6 a b}} / \frac{\sqrt{v \Delta_{x} \Delta_{y}}}{K \sqrt{2 a b^{3}}}$ \\
$t_{b}(x) r_{a}(y)$ & $a=p / 2$ & $y$ & $\lambda\left(f^{\prime}\right)=1-\left|\operatorname{sinc}\left(2 a f^{\prime}\right)\right|$ & $d=b / 0.1780$ & $\frac{\sqrt{v \Delta_{x} \Delta_{y}}}{K \sqrt{6 a b}} / \frac{\sqrt{v \Delta_{x} \Delta_{y}}}{2 K \sqrt{3 a^{2} b \Delta_{x}}}$ \\
\hline $\begin{array}{c}\text { Bitriangular } \\
t_{b}(x) t_{b}(y)\end{array}$ & Sec. 2.2 .3 & $b=p$ & $x$ or $y$ & $\lambda\left(f^{\prime}\right)=1-\operatorname{sinc}^{2}\left(b f^{\prime}\right)$ & $d=b / 0.1780$ & $\frac{\sqrt{2 v \Delta_{x} \Delta_{y}}}{3 K b} / \frac{\sqrt{2 v \Delta_{x} \Delta_{y}}}{\sqrt{3} K b^{2}}$ \\
\hline $\begin{array}{c}\text { Gaussian } \\
g_{\sigma}(x) g_{\sigma}(y)\end{array}$ & Sec. 2.2 .4 & $\sigma=p$ & any & $\lambda\left(f^{\prime}\right)=1-e^{-2 \pi^{2} \sigma^{2} f^{\prime 2}}$ & $d=\sigma / 0.0731$ & $\frac{\sqrt{v \Delta_{x} \Delta_{y}}}{2 \sqrt{2 \pi} K \sigma} / \frac{\sqrt{v \Delta_{x} \Delta_{y}}}{4 \sqrt{\pi} K \sigma^{2}}$ \\
\hline
\end{tabular}

Table 3: Reading guide and summary of the main results. From left to right: window, results on the grid harmonics attenuation, minimum possible half-width (as a function of the pattern pitch $p=1 / f$; note that the support of the Gaussian function is actually larger than $2 \sigma$ ), and metrological quantities (measurement bias, spatial resolution, and measurement resolution) along $x$ - or $y$-direction. Measurement resolution is given first for the phase map, then for the phase derivative map along the considered direction.

\section{Conclusion}

This paper discussed the influence of the analysis window in the grid method to measure displacement and strain fields in experimental mechanics. Four windows used in practice were assessed, namely the birectangular, triangular-rectangular, bitriangular, and Gaussian windows. Our study dealt with several aspects of the method, in the light of its metrological performances.

The main results and a reading guide are gathered in Table 3 . First, we have seen that harmonics of the line profile have to be canceled out. We have established a ranking of the windows with respect to the canceling performance, from the birectangular window to the Gaussian window which performs numerically several orders of magnitude better than the other windows. In addition, the size of the Gaussian window does not require a careful setting since it can take any real value greater than or equal to the grid pitch. We have also proposed an estimation of the measurement bias and of the spatial resolution for each of the windows. Second, we have characterized the propagation of the noise from the imaged grid to the retrieved phases and phase derivatives. Under the simplifying assumption, motivated by the study of the Wigner-Ville transform, that the noise on the phase and its derivatives is stationary, we have found that, at a given spatial resolution, the noise standard deviation, and thus the measurement resolution, was at a comparable level for any of the windows in the phase, and that the Gaussian and bitriangular windows 
gave a similar measurement resolution concerning the phase derivatives, much lower than those in the birectangular and triangular-rectangular cases. However, the stationarity assumption is probably too optimistic concerning the rectangular profile because of the slow decay of its Wigner-Ville transform. On the contrary, the fast decay of the WignerVille transform enforces this assumption in the Gaussian case. The shape of the noise patterns is also smoother in the case of the Gaussian window. This is an important feature for interpreting the strain maps because most details barely emerge from the ground floor, except if a noise reduction procedure is applied beforehand [33]. In our opinion, this discussion advocates for the use of Gaussian windows in the grid method except in situations when very localized phenomena are to be characterized and the noise level is low. Indeed, the Gaussian window has a minimum size which is greater than the bi-triangular one. Therefore, the latter should probably be used in such cases. Windows based on rectangular profiles should not be used, not only because of the slow Wigner-Ville decay and the poor performance of the grid harmonics removal. Indeed, the behavior of their Fourier transforms causes unwanted effects as the one shown in Figure 6. Moreover, contrary to the other studied windows, the Gaussian window is isotropic, which involves an isotropic measurement resolution. This is a desirable feature as we have recently remarked that a rotation of the imaged grid with respect to the pixel grid dramatically reduces aliasing artifacts in some cases [31]. This does not change anything in the analysis presented here, except for the value of $\theta$ in (6) which should be adjusted accordingly. A rotation-invariant window such as the Gaussian one is thus all the more convenient in this case.

Acknowledgement. This work is partially funded by GdR CNRS ISIS (TimEx project).

\section{A Proof of Proposition 1}

Proof. Let $E$ be the expectation of any random variable. Since $n$ is a white noise of variance $v$, we have $E\left(n\left(x_{i}, y_{j}\right) n\left(x_{k}, y_{l}\right)\right)=0$ if $x_{i} \neq x_{k}$ or $y_{j} \neq y_{l}$, and $=v$ otherwise.

By expanding the real and imaginary parts of $\widehat{n}$ and replacing the discrete Riemann sums by integrals:

$$
\begin{aligned}
& \operatorname{Cov}\left(\operatorname{Re}(\widehat{n}(\xi, \eta)), \operatorname{Re}\left(\widehat{n}\left(\xi^{\prime}, \eta^{\prime}\right)\right)\right) \\
& =v \sum_{i, j} w_{\boldsymbol{\sigma}}\left(x_{i}-\xi, y_{j}-\eta\right) w_{\boldsymbol{\sigma}}\left(x_{i}-\xi^{\prime}, y_{j}-\eta^{\prime}\right) \cdot \cos ^{2}\left(2 \pi f x_{i}\right)\left(\Delta_{x} \Delta_{y}\right)^{2} \\
& \quad \simeq v \Delta_{x} \Delta_{y} \iint w_{\boldsymbol{\sigma}}(x-\xi, y-\eta) w_{\boldsymbol{\sigma}}\left(x-\xi^{\prime}, y-\eta^{\prime}\right) \cos ^{2}(2 \pi f x) \mathrm{d} x \mathrm{~d} y
\end{aligned}
$$

Let us define:

$$
I\left(\xi, \eta, \xi^{\prime}, \eta^{\prime}\right)=\iint w_{\boldsymbol{\sigma}}(x-\xi, y-\eta) w_{\boldsymbol{\sigma}}\left(x-\xi^{\prime}, y-\eta^{\prime}\right) \cos ^{2}(2 \pi f x) \mathrm{d} x \mathrm{~d} y
$$


We obtain:

$$
\begin{gathered}
I\left(\xi, \eta, \xi^{\prime}, \eta^{\prime}\right)=\frac{1}{2}\left(\int w_{\sigma_{x}}^{\mathbf{x}}(x-\xi) w_{\sigma_{x}}^{\mathbf{x}}\left(x-\xi^{\prime}\right)(1+\cos (4 \pi f x)) \mathrm{d} x\right) \\
\cdot\left(\int w_{\sigma_{y}}^{\mathbf{y}}(y-\eta) w_{\sigma_{y}}^{\mathbf{y}}\left(y-\eta^{\prime}\right) \mathrm{d} y\right) \\
=\frac{1}{2}\left(w_{\sigma_{x}}^{\mathbf{x}} * w_{\sigma_{x}}^{\mathbf{x}}\left(\xi-\xi^{\prime}\right)+\operatorname{Re}\left(e^{-2 i \pi f\left(\xi+\xi^{\prime}\right)} \int w_{\sigma_{x}}^{\mathbf{x}}(x-\alpha) w_{\sigma_{x}}^{\mathbf{x}}(x+\alpha) e^{-4 i \pi f x} \mathrm{~d} x\right)\right) \\
\cdot w_{\sigma_{y}}^{\mathbf{y}} * w_{\sigma_{y}}^{\mathbf{y}}\left(\eta-\eta^{\prime}\right)
\end{gathered}
$$

where $\alpha=\left(\xi-\xi^{\prime}\right) / 2$.

Since the analysis window is symmetric, we have $w_{\sigma_{x}}^{\mathbf{x}}(x-\alpha)=w_{\sigma_{x}}^{\mathbf{x}}(\alpha-x)$. In addition note that

$$
\int w_{\sigma_{x}}^{\mathbf{x}}(x-\alpha) w_{\sigma_{x}}^{\mathbf{x}}(x+\alpha) e^{-4 i \pi f x} \mathrm{~d} x=\frac{1}{2} \mathcal{W} w_{\sigma_{x}}^{\mathbf{x}}(\alpha, 2 \pi f)
$$

where $\mathcal{W} t_{\sigma}$ is the Wigner-Ville transform of the $1 \mathrm{D}$ window $w_{\sigma_{x}}^{\mathbf{x}}$. Equation (52) follows.

Concerning the imaginary part of the noise:

$$
\begin{aligned}
& \operatorname{Cov}\left(\operatorname{Im}(\widehat{n}(\xi, \eta)), \operatorname{Im}\left(\widehat{n}\left(\xi^{\prime}, \eta^{\prime}\right)\right)\right. \\
& \quad \simeq v \Delta_{x} \Delta_{y} \iint w_{\boldsymbol{\sigma}}(x-\xi, y-\eta) w_{\boldsymbol{\sigma}}\left(x-\xi^{\prime}, y-\eta^{\prime}\right) \sin ^{2}(2 \pi f x) \mathrm{d} x \mathrm{~d} y
\end{aligned}
$$

Thus, the same route as the preceding one gives (53).

Moreover,

$$
\begin{aligned}
& \operatorname{Cov}\left(\operatorname{Re}(\widehat{n}(\xi, \eta)), \operatorname{Im}\left(\widehat{n}\left(\xi^{\prime}, \eta^{\prime}\right)\right)\right. \\
& \quad \simeq v \Delta_{x} \Delta_{y} \iint w_{\boldsymbol{\sigma}}(x-\xi, y-\eta) w_{\boldsymbol{\sigma}}\left(x-\xi^{\prime}, y-\eta^{\prime}\right) \sin (2 \pi f x) \cos (2 \pi f x) \mathrm{d} x \mathrm{~d} y \\
& \quad \simeq \frac{1}{2} v \Delta_{x} \Delta_{y} \iint w_{\boldsymbol{\sigma}}(x-\xi, y-\eta) w_{\boldsymbol{\sigma}}\left(x-\xi^{\prime}, y-\eta^{\prime}\right) \cdot \sin (4 \pi f x) \mathrm{d} x \mathrm{~d} y
\end{aligned}
$$

which gives, in a similar fashion, (54).

\section{B Wigner-Ville transform of some windows}

The Wigner-Ville transform of the rectangular and Gaussian windows can be found in the literature $[7,22]$. However, they sometimes contain typos. For the sake of completeness, we give here the calculation of these transforms. We also give the calculation of the 
Wigner-Ville transform of the triangular windows, which we have not been able to find in the available literature.

A numerical assessment is provided at the following URL:

http://www.loria.fr/ sur/software/VerifWV/

By definition, $\mathcal{W} f(x, \lambda)=\int f(x+\tau / 2) f(x-\tau / 2) e^{-i \tau \lambda} \mathrm{d} \tau$. Since $f$ is symmetric,

$$
\mathcal{W} f(x, \lambda)=2 \int f(\tau+x) f(\tau-x) e^{-2 i \tau \lambda} \mathrm{d} \tau
$$

Since $\mathcal{W} f(-x, \lambda)=\mathcal{W} f(x, \lambda)$, we assume, without loss of generality, that $x \geq 0$.

\section{B.1 Rectangular window}

Here, $f=r_{a}$ in Table 1 . For any $u \in[-a, a]$,

$$
\begin{aligned}
\mathcal{W} f(x, \lambda) & =\frac{1}{2 a^{2}} \int_{x-a}^{a-x} e^{-2 i \tau \lambda} \mathrm{d} \tau \\
& =\frac{1}{2 a^{2} \lambda} \sin (2 \xi(a-x))
\end{aligned}
$$

Thus, for any $x \in \mathbb{R}$,

$$
\mathcal{W} f(x, \lambda)=\frac{1}{2 a^{2} \lambda} \sin (2 \xi(a-|x|)) \mathbb{1}_{[-a, a]}(x)
$$

The value for $\lambda=0$ is given by a Taylor expansion, that is,

$$
\mathcal{W} f(x, 0)=\frac{a-|x|}{a^{2}} \mathbb{1}_{[-a, a]}(x)
$$

\section{B.2 Triangular window}

Here, $f=t_{b}$ in Table 1 :

$$
t_{b}(x)=\frac{b-|x|}{b^{2}} \mathbb{1}_{[-b, b]}(x)
$$

Moreover, if $x \geq b, \mathcal{W} f(x, \lambda)=0$.

First case. $b / 2 \leq x \leq b$. We calculate successively

$$
\begin{aligned}
\mathcal{W} f(x, \lambda) & =2 \int_{x-b}^{b-x} \frac{b-x-\tau}{b^{2}} \frac{b-x+\tau}{b^{2}} e^{-2 i \tau \lambda} \mathrm{d} \tau \\
& =\frac{2}{b^{4}} \int_{x-b}^{b-x}\left((b-x)^{2}-\tau^{2}\right) e^{-2 i \tau \lambda} \mathrm{d} \tau \\
& =\frac{2(b-x)^{2}}{b^{4}} \int_{x-b}^{b-x} e^{-2 i \tau \lambda} \mathrm{d} \tau-\frac{2}{b^{4}} \int_{x-b}^{b-x} \tau^{2} e^{-2 i \tau \lambda} \mathrm{d} \tau
\end{aligned}
$$


On the one hand,

$$
\int_{x-b}^{b-x} e^{-2 i \tau \lambda} \mathrm{d} \tau=\sin (2 \xi(b-x)) / \lambda
$$

On the other hand,

$$
\begin{aligned}
\int_{x-b}^{b-x} \tau^{2} e^{-2 i \tau \lambda} \mathrm{d} \tau & =\frac{(b-x)^{2}}{\lambda} \sin (2 \lambda(b-x))+\frac{1}{i \xi} \int_{x-b}^{b-x} \tau e^{-2 i \tau \lambda} \mathrm{d} \tau \\
& =\frac{(b-x)^{2}}{\lambda} \sin (2 \lambda(b-x)) \\
+\frac{1}{i \lambda}\left(\frac{x-b}{i \lambda}\right. & \left.\cos (2 \lambda(b-x))+\frac{1}{2 i \lambda^{2}} \sin (2 \lambda(b-x))\right) \\
& =\frac{(b-x)^{2}}{\lambda} \sin (2 \xi(b-x)) \\
+\frac{b-x}{\lambda^{2}} & \cos (2 \lambda(b-x))-\frac{1}{2 \lambda^{3}} \sin (2 \lambda(b-x))
\end{aligned}
$$

Thus,

$$
\mathcal{W} f(x, \lambda)=\frac{2}{b^{4} \lambda^{2}}\left(-(b-x) \cos (2 \lambda(b-x))+\frac{1}{2 \lambda} \sin (2 \lambda(b-x))\right)
$$

Second case. $0 \leq x \leq b / 2$. We calculate successively

$$
\begin{aligned}
\mathcal{W} f(x, \lambda)= & 2 \int_{x-b}^{-x} \frac{b+x+\tau}{b^{2}} \frac{b-x+\tau}{b^{2}} e^{-2 i \tau \lambda} \mathrm{d} \tau+2 \int_{-x}^{x} \frac{b-x-\tau}{b^{2}} \frac{b-x+\tau}{b^{2}} e^{-2 i \tau \lambda} \mathrm{d} \tau \\
& +2 \int_{x}^{b-x} \frac{b-x-\tau}{b^{2}} \frac{b+x-\tau}{b^{2}} e^{-2 i \tau \lambda} \mathrm{d} \tau \\
= & \frac{2}{b^{4}} \int_{-x}^{x}\left((b-x)^{2}-\tau^{2}\right) e^{-2 i \tau \lambda} \mathrm{d} \tau \\
& +\frac{4}{b^{4}} \int_{x}^{b-x}\left((b-\tau)^{2}-x^{2}\right) \cos (2 \tau \lambda) \mathrm{d} \tau \\
= & \frac{2(b-x)^{2}}{b^{4}} \int_{-x}^{x} e^{-2 i \tau \lambda} \mathrm{d} \tau-\frac{2}{b^{4}} \int_{-x}^{x} \tau^{2} e^{-2 i \tau \lambda} \mathrm{d} \tau \\
& +\frac{4}{b^{4}} \int_{x}^{b-x}(b-\tau)^{2} \cos (2 \tau \lambda) \mathrm{d} \tau-\frac{4 x^{2}}{b^{4}} \int_{x}^{b-x} \cos (2 \tau \lambda) \mathrm{d} \tau
\end{aligned}
$$

Now,

$$
\begin{gathered}
\int_{-x}^{x} e^{-2 i \tau \lambda} \mathrm{d} \tau=\sin (2 \lambda x) / \lambda \\
\int_{-x}^{x} \tau^{2} e^{-2 i \tau \lambda} \mathrm{d} \tau=\frac{x^{2}}{\lambda} \sin (2 \lambda x)+\frac{x}{\lambda^{2}} \cos (2 \lambda x)-\frac{1}{2 \lambda^{3}} \sin (2 \lambda x)
\end{gathered}
$$




$$
\begin{gathered}
\int_{x}^{b-x} \cos (2 \tau \lambda) \mathrm{d} \tau=\frac{1}{2 \lambda}(\sin (2 \lambda(b-x))-\sin (2 \lambda x)) \\
\int_{x}^{b-x}(b-\tau)^{2} \cos (2 \tau \lambda) \mathrm{d} \tau=\frac{x^{2}}{2 \lambda} \sin (2(b-x) \lambda)-\frac{(b-x)^{2}}{2 \lambda} \sin (2 \lambda x)+\frac{1}{\lambda} \int_{x}^{b-x}(b-\tau) \sin (2 \tau \lambda) \mathrm{d} \tau \\
=\frac{x^{2}}{2 \lambda} \sin (2(b-x) \lambda)-\frac{(b-x)^{2}}{2 \lambda} \sin (2 \lambda x) \\
-\frac{x}{2 \lambda^{2}} \cos (2 \lambda(b-x))+\frac{(b-x)}{2 \lambda^{2}} \cos (2 \lambda x)-\frac{1}{2 \lambda^{2}} \int_{x}^{b-x} \cos (2 \tau \lambda) \mathrm{d} \tau \\
=\frac{x^{2}}{2 \lambda} \sin (2(b-x) \lambda)-\frac{(b-x)^{2}}{2 \lambda} \sin (2 \lambda x)-\frac{x}{2 \lambda^{2}} \cos (2 \lambda(b-x)) \\
+\frac{(b-x)}{2 \lambda^{2}} \cos (2 \lambda x)-\frac{1}{4 \lambda^{3}}(\sin (2 \lambda(b-x))-\sin (2 \lambda x))
\end{gathered}
$$

Consequently,

$$
\begin{aligned}
\mathcal{W} f(x, \lambda)=\frac{2}{b^{4} \lambda^{2}}((b-2 x) \cos (2 \lambda x)-x \cos (2 \lambda(b-x)) & \\
& \left.-\frac{1}{2 \lambda} \sin (2 \lambda(b-x))+\frac{1}{\lambda} \sin (2 \lambda x)\right)
\end{aligned}
$$

Conclusion. For any $x \in \mathbb{R}$, the Wigner-Ville transform of the triangle function writes

$$
\begin{aligned}
& \mathcal{W} f(x, \lambda)=\frac{2}{b^{4} \lambda^{2}}\left[\left(-(b-|x|) \cos (2 \lambda(b-|x|))+\frac{1}{2 \lambda} \sin (2 \lambda(b-|x|))\right) \mathbb{1}_{[-b, b]}(x)\right. \\
& \left.\quad+2 \cos (\lambda b)\left((b-2|x|) \cos (\lambda(b-2|x|))-\frac{1}{\lambda} \sin (\lambda(b-2|x|))\right) \mathbb{1}_{[-b / 2, b / 2]}(x)\right]
\end{aligned}
$$

The value for $\lambda=0$ is given by a Taylor expansion:

$$
\mathcal{W} f(x, 0)=\frac{8(b-|x|)^{3}}{3 b^{4}} \mathbb{1}_{[-b, b]}(x)-\frac{4(b-2|x|)^{3}}{3 b^{4}} \mathbb{1}_{[-b / 2, b / 2]}(x)
$$

\section{B.3 Gaussian window}

Note that generalized Gaussian functions give positive Wigner-Ville transform, and only them [10]. The Wigner-Ville transform of a Gaussian function writes as follows:

$$
\begin{aligned}
\mathcal{W} f(x, \lambda) & =\frac{1}{\pi \sigma^{2}} \int e^{-\left((\tau+x)^{2}-(\tau-x)^{2}\right) /\left(2 \sigma^{2}\right)} e^{-2 i \tau \lambda} \mathrm{d} \tau \\
& =\frac{e^{-x^{2} / \sigma^{2}}}{\pi \sigma^{2}} \int e^{-\tau^{2} / \sigma^{2}} e^{-2 i \tau \lambda} \mathrm{d} \tau \\
& =\frac{1}{\sqrt{\pi} \sigma} e^{-x^{2} / \sigma^{2}-\lambda^{2} \sigma^{2}}
\end{aligned}
$$

since the Fourier transform of $e^{-x^{2} / \sigma^{2}}$ is $\sqrt{\pi} \sigma e^{-f^{2} \sigma^{2} / 4}$. 


\section{Noise derivative with a birectangular or triangular - rectangular window}

Let $X(t)$ be a stationary random process of covariance function $C(u)$. For any $\varepsilon>0$, the covariance of $\frac{X(t+\varepsilon)-X(t)}{\varepsilon}$ writes as follows.

$$
\begin{aligned}
& \operatorname{Cov}\left(\frac{X(t+\varepsilon)-X(t)}{\varepsilon}, \frac{X\left(t^{\prime}+\varepsilon\right)-X\left(t^{\prime}\right)}{\varepsilon}\right) \\
&=\frac{1}{\varepsilon^{2}}\left(\operatorname{Cov}\left(X(t+\varepsilon), X\left(t^{\prime}+\varepsilon\right)\right)+\operatorname{Cov}\left(X(t), X\left(t^{\prime}\right)\right)-\operatorname{Cov}\left(X(t+\varepsilon), X\left(t^{\prime}\right)\right)\right. \\
&\left.-\operatorname{Cov}\left(X(t), X\left(t^{\prime}-\varepsilon\right)\right)\right) \\
&\left.=\frac{1}{\varepsilon^{2}}(C(u)-C(u+\varepsilon)-C(u-\varepsilon))\right)
\end{aligned}
$$

with $u=t-t^{\prime}$.

When the second derivative of the covariance function $C$ exists, this latest term converges to $-C^{\prime \prime}(u)$ as $\varepsilon$ tends to 0 . This makes it possible to define the mean-square derivative $X^{\prime}(t)$ of the random process $X$ at $t$ as soon as the second derivative exists; the covariance function of $X(t)$ being $-C^{\prime \prime}(t)$.

This obviously generalizes to stationary random fields (see, e.g., [1]). In the case of rectangular windows, the second derivatives do not exist. However, actual computations do not depend on the mean-square derivatives, but instead on a finite difference scheme.

If a central difference scheme of step $\Delta_{x}=1$ pixel is used to estimate the derivatives,

$$
\begin{aligned}
\operatorname{Cov}\left(\frac{X\left(t+\Delta_{x}\right)-X\left(t-\Delta_{x}\right)}{2 \Delta_{x}}, \frac{X\left(t^{\prime}+\Delta_{x}\right)-X\left(t^{\prime}-\Delta_{x}\right)}{2 \Delta_{x}}\right) \\
=\frac{1}{4 \Delta_{x}^{2}}\left(C\left(u+2 \Delta_{x}\right)-2 C(u)+C\left(u+2 \Delta_{x}\right)\right)
\end{aligned}
$$

which is the opposite of the second derivative of $C$ (in the sense of the central difference scheme), denoted here by $-\Delta^{2} C$. The problem is to compute the noise covariance in the case of birectangular and triangular-rectangular windows, thus to compute the $-\Delta^{2} r_{a} * r_{a}(u)=-\frac{1}{2} \Delta^{2} \mathcal{W} r_{a}(u / 2,0)$.

A straightforward calculation gives

$$
\begin{aligned}
-\Delta^{2} r_{a} * & r_{a}(u)=\frac{-1}{8 a^{2} \Delta_{x}^{2}}\left(\left(\Delta_{x}-|2 a+t| / 2\right) \mathbb{1}_{\left[-2 a-2 \Delta_{x},-2 a+2 \Delta_{x}\right]}(t)\right. \\
& \left.-2\left(\Delta_{x}-|t| / 2\right) \mathbb{1}_{\left[-2 \Delta_{x}, 2 \Delta_{x}\right]}(t)+\left(\Delta_{x}-|2 a-t| / 2\right) \mathbb{1}_{\left[2 a-2 \Delta_{x}, 2 a+2 \Delta_{x}\right]}(t)\right)
\end{aligned}
$$




\section{References}

[1] P. Abrahamsen. A review of Gaussian random fields and correlation functions. Technical report, Norwegian Computing Center, Oslo, 1997.

[2] S. Avril, E. Ferrier, A. Vautrin, P. Hamelin, and Y. Surrel. A full-field optical method for the experimental analysis of reinforced concrete beams repaired with composites. Composites Part A: Applied Science and Manufacturing, 35(7-8):873$884,2004$.

[3] C. Badulescu, M. Grédiac, and J.-D. Mathias. Investigation of the grid method for accurate in-plane strain measurement. Measurement Science and Technology, 20(9):095102, 2009.

[4] C. Badulescu, M. Grédiac, J.-D. Mathias, and D. Roux. A procedure for accurate one-dimensional strain measurement using the grid method. Experimental Mechanics, 49(6):841-854, 2009.

[5] X. Balandraud, N. Barrera, P. Biscari, M. Grédiac, and G. Zanzotto. Strain intermittency in shape memory alloys. Physical Review B, 91(17):174111, 2015.

[6] A. Chrysochoos and Y. Surrel. Basics of metrology and introduction to techniques. In M. Grédiac and F. Hild, editors, Full-field measurements and identification in solid mechanics, pages 1-29. Wiley, 2012.

[7] L. Cohen. Time-frequency analysis. Prentice-Hall, 1995.

[8] X. Dai, H. Xie, and Q. Wang. Geometric phase analysis based on the windowed Fourier transform for the deformation field measurement. Optics and Laser Technology, 58:119-127, 2014.

[9] D. Delpueyo, M. Grédiac, X. Balandraud, and C. Badulescu. Investigation of martensitic microstructures in a monocrystalline $\mathrm{Cu}$-Al-Be shape memory alloy with the grid method and infrared thermography. Mechanics of Materials, 45(1):34-51, 2012 .

[10] P. Flandrin. Separability, positivity, and minimum uncertainty in time-frequency energy distributions. Journal of Mathematical Physics, 39(8):4016-4040, 1998.

[11] M. Grédiac and F. Sur. Effect of sensor noise on the resolution and spatial resolution of displacement and strain maps estimated with the grid method. Strain, 50(1):1-27, 2014 .

[12] M. Grédiac, F. Sur, and B. Blaysat. The grid method for in-plane displacement and strain measurement: a review and analysis. Strain, 2016. To be published.

[13] M. Grédiac and E. Toussaint. Studying the mechanical behaviour of asphalt mixtures with the grid method. Strain, 49(1):1-15, 2013. 
[14] F.J. Harris. On the use of windows for harmonic analysis with the discrete Fourier transform. Proceedings of the IEEE, 66:51-83, 1978.

[15] G.E. Healey and R. Kondepudy. Radiometric CCD camera calibration and noise estimation. IEEE Transactions on Pattern Analysis and Machine Intelligence, 16(3):267-276, 1994.

[16] F. Hlawatsch and G.F. Boudreaux-Bartels. Linear and quadratic time-frequency signal representations. IEEE Signal Processing Magazine, 9(2):21-67, 1992.

[17] L. Huang, Q. Kemao, B. Pan, and A.K. Asundi. Comparison of Fourier transform, windowed Fourier transform, and wavelet transform methods for phase extraction from a single fringe pattern in fringe projection profilometry. Optics and Lasers in Engineering, 48:141-148, 2010.

[18] J.M. Huntley. Automated fringe pattern analysis in experimental mechanics: A review. The Journal of Strain Analysis for Engineering Design, 33(2):105-125, 1998.

[19] JCGM member organizations. International vocabulary of metrology. basic and general concepts and associated terms (VIM), 2008.

[20] Q. Kemao. Windowed Fourier transform for fringe pattern analysis. Applied Optics, 43(13):2695-2702, 2004.

[21] Q. Kemao. Two-dimensional windowed Fourier transform for fringe pattern analysis: Principles, applications and implementations. Optics and Lasers in Engineering, 45(2):304-317, 2007.

[22] S. Mallat. A wavelet tour of signal processing (2nd edition). Academic Press, 1999.

[23] J. Molimard and Y. Surrel. Grid method, moiré and deflectometry. In M. Grédiac and F. Hild, editors, Full-field Measurements and Identification in Solid Mechanics, pages 61-89. Wiley, 2012.

[24] R. Moulart, R. Rotinat, F. Pierron, and G. Lerondel. On the realization of microscopic grids for local strain measurement by direct interferometric photolithography. Optics and Lasers in Engineering, 45(12):1131-1147, 2007.

[25] F. Murthagh, J.L. Starck, and A. Bijaoui. Image restoration with noise suppression using a multiresolution support. Astronomy and astrophysics, 112:179-189, 1995.

[26] A. W. Peevers. A real time 3D signal analysis/synthesis tool based on the short time Fourier transform. Technical report, Department of Electrical Engineering, University of California, Berkeley, USA, 2004.

[27] F. Pierron, H. Zhu, and C. Siviour. Beyond Hopkinson's bar. Philosophical Transactions of the Royal Society A: Mathematical, Physical and Engineering Sciences, 372(2023):20130195, 2014. 
[28] R. Moutou Pitti, C. Badulescu, and M. Grédiac. Characterization of a cracked specimen with full-field measurements: direct determination of the crack tip and energy release rate calculation. International Journal of Fracture, 187(1):109-121, 2014 .

[29] P. L. Reu, E. Quintana, and K. Lon. Using sampling moiré to extract displacement information from X-ray images of molten salt batteries. In Conference proceedings of the Society for Experimental Mechanics series, volume 3, pages 331-336, 2014.

[30] S. Ri, T. Muramatsu, M. Saka, K. Nanbara, and D. Kobayashi. Noncontact deflection distribution measurement for large-scale structures by advanced image processing technique. Materials Transactions, 53(2):323-329, 2012.

[31] F. Sur, B. Blaysat, and M. Grédiac. Determining displacement and strain maps immune from aliasing effect with the grid method. 2016. Submitted for publication.

[32] F. Sur and M. Grédiac. Towards deconvolution to enhance the grid method for in-plane strain measurement. AIMS Inverse Problems and Imaging, 8(1):259-291, 2014.

[33] F. Sur and M. Grédiac. On noise reduction in strain maps obtained with the grid method by averaging images affected by vibrations. Optics and Lasers in Engineering, 66:210-222, 2015.

[34] Y. Surrel. Design of algorithms for phase measurements by the use of phase stepping. Applied Optics, 35(1):51-60, 1996.

[35] Y. Surrel. Additive noise effect in digital phase detection. Applied Optics, 36(1):271$276,1997$.

[36] Y. Surrel. Photomechanics, volume 77 of Topics in Applied Physics, chapter Fringe analysis, pages 55-102. Springer, 2000.

[37] L. Wittevrongel, P. Lava, S.V. Lomov, and D. Debruyne. A self adaptive global digital image correlation algorithm. Experimental Mechanics, 55(2):361-378, 2015. 